\title{
GLOBAL AVERAGING AND PARAMETRIC RESONANCES IN DAMPED SEMILINEAR WAVE EQUATIONS
}

\author{
S. ZELIK \\ Universität Stuttgart \\ Institut für Analysis, Dynamik und Modelierung \\ Pfaffenwaldring 57 \\ 70569 Stuttgart, Germany
}

\begin{abstract}
The longtime behavior of solutions to a semilinear damped wave equation in a three-dimensional bounded domain with the nonlinearity rapidly oscillating in time $(f=f(\varepsilon, u, t / \varepsilon))$ is studied. It is proved that (under natural assumptions) the behavior of solutions whose initial energy is not very large can be described in terms of global (uniform) attractors $\mathcal{A}_{\varepsilon}$ of the corresponding dynamical processes and that these attractors tend as $\varepsilon \rightarrow 0$ to the global attractor $\mathcal{A}_{0}$ of the corresponding averaged system. We also give the detailed description of these attractors in the case where the limit attractor $\mathcal{A}_{0}$ is regular.

Moreover, we give explicit examples of semilinear hyperbolic equations where the uniform attractor $\widehat{\mathcal{A}_{\varepsilon}}$ (for the initial data belonging to the whole energy phase space) contains the irregular resonant part which tends to infinity as $\varepsilon \rightarrow 0$ and formulate the additional restrictions on the nonlinearity $f$ which guarantee that this part is absent.
\end{abstract}

\section{INTRODUCTION.}

We consider the following semilinear damped hyperbolic equation in a bounded smooth three-dimensional domain $\Omega \subset \mathbb{R}^{3}$ :

$$
\left\{\begin{array}{l}
\partial_{t}^{2} u+\gamma \partial_{t} u-\Delta_{x} u+\lambda_{0} u+f(\varepsilon, u, t / \varepsilon)=g \\
\left.u\right|_{t=\tau}=u_{\tau},\left.\quad \partial_{t} u\right|_{t=\tau}=u_{\tau}^{\prime},\left.\quad \partial_{n} u\right|_{\partial \Omega}=0 .
\end{array}\right.
$$

Here $\gamma$ and $\lambda_{0}$ are fixed positive constants, the external forces $g \in L^{2}(\Omega), \varepsilon>0$ is a small parameter and the nonlinear interaction function $f(\varepsilon, u, z)$ is smooth enough with respect to $u$ and $\varepsilon$ and is almost-periodic with respect to $z$ (see Section 1 for the precise conditions).

As usual, we complete the family of problems (0.1) at $\varepsilon=0$ by the following averaged equation:

$$
\partial_{t}^{2} \bar{u}+\gamma \partial_{t} \bar{u}-\Delta_{x} \bar{u}+\lambda_{0} \bar{u}+\bar{f}(\bar{u})=g
$$

1991 Mathematics Subject Classification. 35B40, 35B45, 35L30.

Key words and phrases. Singularly perturbed damped wave equation, global averaging, regular attractors.

Typeset by $\mathcal{A}_{\mathcal{M}} \mathcal{S}-\mathrm{T}_{\mathrm{E}} \mathrm{X}$ 
where $\bar{f}(u)$ is the average of the almost-periodic function $f(0, u, z)$. We also impose the standard dissipativity and growth restrictions on the average $\bar{f}(u)$ which guarantee the global existence and dissipativity of solutions of equation (0.2) in the energy space $E^{1}(\Omega):=H^{2}(\Omega) \cap\left\{\left.\partial_{n} u\right|_{\partial \Omega}=0\right\} \times H^{1}(\Omega)$ (the critical cubic rate of growth of $\bar{f}$ is also allowed, see Section 1$)$. It is also worth noting that no growth and dissipativity assumptions on the nonlinearities $f(\varepsilon, u, z)$ for positive $\varepsilon$ are imposed.

The long-time behavior of solutions to (0.1) in the autonomous case is usually described in terms of global attractors of the dynamical systems associated with the problem under consideration, see [3], [19], [29] and references therein.

The case of non-autonomous equations is essentially less understood. In fact, up to the moment, there are several natural approaches to extend the attractors theory to the non-autonomous case. One of them is based on a reduction of the non-autonomous dynamical process to the autonomous one, using the skew-product technique. The realization of this approach leads to the so-called uniform attractor $\mathcal{A}_{\varepsilon}$ of equation (0.1) which is independent of $t$ and is uniform with respect to all the nonlinearities $\phi(\varepsilon, u, t / \varepsilon)$ belonging to the hull $\mathcal{H}(f)$ of the initial nonlinearity $f$, see [8], [20]. The alternative approach interprets the attractor of the non-autonomous equation (0.1) as a non-autonomous set as well: $\mathcal{A}_{f}(t), t \in \mathbb{R}$, see e.g. [12], [22].

The homogenization problems for individual solutions of evolution equations with rapidly oscillating spatial and temporal terms were investigated in [2], [4], [5], [24], [28], [36] (see also references therein).

The homogenization of attractors were also studied by many authors. See, e.g. [6], [27] for attractors of reaction-diffusion and hyperbolic equations in nonhomogenized spatially-periodic media with asymptotic degeneracy. The case of regular spatially almost-periodic media was considered in [13]. The homogenization aspects for the evolution problems with spatially rapidly oscillations in subordinated terms (i.e. for $f=f(x / \varepsilon, u)$ or $g=g(x / \varepsilon)$ ) were considered in [16] and [17]. The temporal averaging of uniform attractors for evolutionary problems were studied in [21] (for the case of the nonlinear wave equation with rapidly oscillating in time external forces) and in [33] (for the case of singularly perturbed reactiondiffusion system with rapidly oscillating external forces). The non-autonomous regular attractors for reaction-diffusion equations with rapidly oscillating in time nonlinearities were investigated in [14]. The homogenization of trajectory attractors associated with ill-posed evolutionary mathematical physics equations (such as 3D Navier-Stokes equations, damped wave equations with supercritical nonlinearities, etc.) were studied in [8] and [11].

In the present paper, we carry out a detailed analysis of problems related with the local and global averaging of the solutions of semilinear hyperbolic equations (0.1). In particular, we prove (in Section 2) that the dissipativity of the averaged system $(0.2)$ in the energy space $E^{1}(\Omega)$ implies the existence of a global bounded solution for problem $(0.1)$ with the initial data $\xi^{\tau}:=\left(u_{\tau}, u_{\tau}^{\prime}\right)$ belonging to a large ball $B_{R_{0}(\varepsilon)}$ (if $\varepsilon>0$ is small enough) where the radius $R_{0}(\varepsilon)$ tends to infinity as $\varepsilon \rightarrow 0$.

We also establish (in Section 3) that the longtime behavior of the solutions to equation (0.1) with the initial data belonging to $B_{R_{0}(\varepsilon)}$ can be described in terms of the uniform attractor $\mathcal{A}_{\varepsilon}$ of the corresponding dynamical process, and that the attractors $\mathcal{A}_{\varepsilon}$ are uniformly bounded in $E^{1}(\Omega)$ and tend (as $\varepsilon \rightarrow 0$ ) to the global 
attractor $\mathcal{A}_{0}$ of the limit autonomous problem (0.2) (in the sense of the upper semicontinuity in $\left.E^{1}(\Omega)\right)$.

Moreover, under the additional generic assumption that the limit attractor $\mathcal{A}_{0}$ is regular, we provide a detailed description (in the spirit of [14]) of the pullback attractor $\mathcal{A}_{f}(\tau)$ of equation (0.1) on the ball $B_{R_{0}(\varepsilon)}$ for small positive $\varepsilon$. In particular, we establish that in this case, equation (0.1) possesses only a finite number of different almost periodic solutions (which are close to the equilibria of the averaged system) and any other solution of this equation (belonging to the ball $B_{R_{0}(\varepsilon)}$ ) tends as $t \rightarrow \infty$ to one of these almost-periodic solution, see Section 5 . Furthermore, the pullback attractor $\mathcal{A}_{f}(\tau)$ has a regular structure (i.e., it is a finite collection of the finite-dimensional unstable manifolds of the almost-periodic solution mentioned above), attracts exponentially and uniformly with respect to $\varepsilon$ and $\tau$ the images of bounded subsets $B \subset B_{R_{0}(\varepsilon)}$ and tends to the limit global attractor $\mathcal{A}_{0}$ as $\varepsilon \rightarrow 0$ in the sense of upper and lower semicontinuity in $E^{1}(\Omega)$, see Section 5 for the details.

We however note that, in contrast to the case of parabolic equations or hyperbolic equations with rapidly oscillating in time external forces considered in the previous papers, see e.g., [8] [14] and [34], in our case, the uniform attractor $\widehat{\mathcal{A}_{\varepsilon}}$ of equation $(0.1)$ in the whole phase space $E^{1}(\Omega)$ (i.e., the initial data outside of the ball $B_{R_{0}(\varepsilon)}$ is allowed), if it exists, it does not necessarily coincide with the uniform attractor $\mathcal{A}_{\varepsilon}$ described above and it does not necessarily tend to the averaged attractor $\mathcal{A}_{0}$ as $\varepsilon \rightarrow 0$. Indeed, we give (in Section 7) an example of equation of the form (0.1) whose attractor $\widehat{\mathcal{A}_{\varepsilon}}$ consists of two parts. The first one $\left(\mathcal{A}_{\varepsilon}\right)$ is regular, has a large basin of attraction (which contains at least the ball $B_{R_{0}(\varepsilon)}$ ) and tends to the limit averaged attractor $\mathcal{A}_{0}$ as $\varepsilon \rightarrow 0$. The second part is, however, irregular (chaotic) and tends to infinity as $\varepsilon \rightarrow 0$, see Examples 7.4 and 7.7. The existence of the irregular part of the attractor $\widehat{\mathcal{A}_{\varepsilon}}$ in our example can be explained in terms of the so-called nonlinear parametric resonance phenomena (see Section 7) which is typical for hyperbolic equations and, therefore, we belive that the irregular part of the attractor is nonempty for more or less general equations of the form (0.1) where the leading part of the nonlinearity contains rapid oscillations in time.

Nevertheless, we introduce a rather wide class of equations of the form (0.1) (the so-called subordinated oscillations), for which we prove that the irregular part of the attractor is empty and

$$
\widehat{\mathcal{A}_{\varepsilon}}=\mathcal{A}_{\varepsilon}
$$

exactly as in the case of reaction-diffusion equations.

The paper is organized as follows. The precise formulation of our assumptions on the functions $f(\varepsilon, u, z)$ and some auxiliary results, which are of fundamental significance for what follows, are given in Section 1.

The local averaging of equation (0.1) (on a finite interval of time) is considered in Section 2. Moreover, based on this result, we also establish there the existence of global bounded solutions for problem (0.1), whose initial energy is not very large.

The uniform and pullback attractors for equation (0.1) (with the initial data belonging to $\left.B_{R_{0}(\varepsilon)}\right)$ are constructed in Section 3 and their convergence to the limit global attractor $\mathcal{A}_{0}$ is there verified.

Section 4 is devoted to study the behavior of the solutions to equation (0.1) in a small neighborhood of the hyperbolic equilibrium $z_{0}(x)$ of the averaged equation (0.2), which is necessary for the regular attractors theory. 
In Section 5 we give a detailed description of the pullback attractors $\mathcal{A}_{f}(\tau)$ in the case where the limit attractor $\mathcal{A}_{0}$ is regular. In particular, the upper and lower semicontinuity of attractors $\mathcal{A}_{\varepsilon}$ at $\varepsilon=0$ is established.

In Section 6, we formulate the additional assumptions on the nonlinearity $f$ which guarantee equality (0.3).

The obtained results are illustrated by several concrete examples of equations of the form (0.1) which are given in Section 7.

Finally, several auxiliary estimates for the linear hyperbolic equations are obtained in the Appendix.

Acknowledgements. This research is partially supported by the INTAS project no. 00-899, CRDF grant RM1-2343-MO-02 and Alexander von Humboldt foundation. The author is also grateful to A.Ilyin and A.Liapin for the stimulating discussions.

\section{$\S 1$ MAIN ASSUMPTIONS AND PRELIMINARY RESUlTS.}

In this section we formulate our assumptions on the nonlinear interaction function $f(\varepsilon, u, t / \varepsilon)$, recall some known facts on almost-periodic functions and prepare the technical tools for the next sections. To be precise, we assume from now on that, for every $\varepsilon \geq 0$ and $z \in \mathbb{R}$, the functions $f(\varepsilon, \cdot, z), f_{u}^{\prime}(\varepsilon, \cdot, z), f_{u}^{\prime \prime}(\varepsilon, \cdot, z)$ and $f_{u}^{\prime \prime \prime}(\varepsilon, \cdot, z)$ belong to $C(\mathbb{R})$ and satisfy the following estimate:

$$
|f(\varepsilon, u, z)|+\left|f_{u}^{\prime}(\varepsilon, u, z)\right|+\left|f_{u}^{\prime \prime}(\varepsilon, u, z)\right|+\left|f_{u}^{\prime \prime \prime}(\varepsilon, u, z)\right| \leq Q(|u|),
$$

for some monotonic function $Q$ which is independent of $\varepsilon$ and $z$. We also assume that $f(\varepsilon, u, z)$ tends to $f(0, u, z)$ as $\varepsilon \rightarrow 0$ in the following sense:

$$
\begin{aligned}
|f(\varepsilon, u, z)-f(0, u, z)|+\left|f_{u}^{\prime}(\varepsilon, u, z)-f_{u}^{\prime}(0, u, z)\right|+ & \\
& +\left|f_{u}^{\prime \prime}(\varepsilon, u, z)-f_{u}^{\prime \prime}(0, u, z)\right| \leq C_{R} \varepsilon, \quad \forall|u| \leq R
\end{aligned}
$$

and every $\varepsilon$ and $z$ where the constant $C_{R}$ is independent of $z$ and $\varepsilon$.

Our next assumption is that, for every $\varepsilon \geq 0$, the functions $z \rightarrow f(\varepsilon, u, z)$, $f_{u}^{\prime}(\varepsilon, u, z), f_{u}^{\prime \prime}(\varepsilon, u, z)$ and $f_{u}^{\prime \prime \prime}(\varepsilon, u, z)$ are almost-periodic as functions with values in the space $C_{l o c}(\mathbb{R})$. We recall that a function $\phi(u, z)$ is almost-periodic as a $C_{l o c}(\mathbb{R})$ valued function if and only if $\phi(u, z)$ is an almost-periodic real-valued function (in the Bohr sense, see e.g., [23]) for every fixed $u \in \mathbb{R}$, and it is uniformly continuous with respect to $u$ belonging to bounded subsets and $z \in \mathbb{R}$, i.e., for every $R>0$ there exists a monotone function $\alpha_{R}: \mathbb{R}_{+} \rightarrow \mathbb{R}$ such that $\lim _{\varepsilon \rightarrow 0} \alpha_{R}(\varepsilon)=0$ and

$$
\left|\phi\left(u_{1}, z\right)-\phi\left(u_{2}, z\right)\right| \leq \alpha_{R}\left(\left|u_{1}-u_{2}\right|\right), \forall u_{1}, u_{2} \in \mathbb{R},\left|u_{i}\right| \leq R \text { and every } z \in \mathbb{R}
$$

see, e.g., [14] and [24] for the details. We also recall that every almost-periodic with respect to $z$ function $z \rightarrow \phi(u, z)$ possesses the average

$$
\mathbb{M}(\phi)(u):=\lim _{T \rightarrow \infty} \frac{1}{T} \int_{t}^{t+T} \phi(u, z) d z
$$

where the limit is uniform with respect to $t \in \mathbb{R}$. Moreover, if, in addition, $\phi$ is almost-periodic as a $C_{l o c}(\mathbb{R})$-valued function (i.e., (1.3) is satisfied), then the limit 
(1.4) is uniform with respect to $u$ belonging to bounded subsets as well. Namely, for every $R \in \mathbb{R}_{+}$, there exists a monotone function $\alpha_{R}: \mathbb{R}_{+} \rightarrow \mathbb{R}$ such that $\lim _{\varepsilon \rightarrow 0} \alpha_{R}(\varepsilon)=0$ and

$$
\left|\mathbb{M}(\phi)(u)-\frac{1}{T} \int_{t}^{t+T} \phi(u, z) d z\right| \leq \alpha_{R}(1 / T)
$$

for every $u$ such that $|u| \leq R$ and every $t \in \mathbb{R}$, see [24] for the details.

We now define, for every $\varepsilon>0$, the function

$$
f_{\varepsilon}(u, t):=f(\varepsilon, u, t / \varepsilon)
$$

and complete this family of functions as $\varepsilon=0$ by

$$
f_{0}(u)=\bar{f}(u):=\mathbb{M}(f(0, u, z))(u) .
$$

We will consider the function $\bar{f}$ as the average of the functions (1.6).

We do not impose any growth or dissipativity assumptions on the functions $f_{\varepsilon}$, which guarantee the global solvability of equation (0.1) for $\varepsilon>0$. In contrast to this, the global solvability of the averaged equation (0.1) (with $\varepsilon=0$ ) is crucial for our method, so we need the average $\bar{f}$ to satisfy the following additional assumptions:

$$
\left\{\begin{array}{l}
\text { 1. } \quad \bar{f} \in C^{3}(\mathbb{R}), \\
\text { 2. } \quad\left|\bar{f}^{\prime \prime \prime}(u)\right| \leq C, \quad \forall u \in \mathbb{R}, \\
\text { 3. } \quad \liminf _{|u| \rightarrow \infty} \bar{f}^{\prime}(u) \geq 0 .
\end{array}\right.
$$

We start our exposition with the following lemma which clarifies in what sense the functions $f_{\varepsilon}(u, t)$ tend to $\bar{f}(u)$ as $\varepsilon \rightarrow 0$.

Lemma 1.1. Let the above assumptions hold and let $\varphi_{\varepsilon}$ and $\bar{\varphi}$ be one of the following functions $f_{\varepsilon}, \partial_{u} f_{\varepsilon}, \partial_{u}^{2} f_{\varepsilon}$ and $\bar{f}, \partial_{u} \bar{f}, \partial_{u}^{2} \bar{f}$ respectively. Then, for every $R>0$, the following estimate holds:

$$
\left|\int_{t}^{t+\tau}\left[\varphi_{\varepsilon}(u, t)-\bar{\varphi}(u)\right] d t\right| \leq \alpha_{R}(\varepsilon), \quad \forall|u| \leq R, t \in \mathbb{R}, \quad \tau \in[0,1], \varepsilon \geq 0
$$

where the monotonic function $\alpha_{R}(\varepsilon)$ is independent of $u, t$ and $\tau$ and satisfies the condition $\lim _{\varepsilon \rightarrow 0} \alpha_{R}(\varepsilon)=0$.

Proof. Thanks to (1.2), it is sufficient to estimate the following term

$$
\int_{t}^{t+\tau}[f(0, u, t / \varepsilon)-\bar{f}(u)] d t=\varepsilon \int_{t / \varepsilon}^{t / \varepsilon+\tau / \varepsilon}[f(0, u, z)-\bar{f}(z)] d z
$$

and its first and second derivatives with respect to $u$. But, due to our assumptions, the function $z \rightarrow f(0, u, z)$ and its first and second derivative with respect to $u$ are almost-periodic as $C_{l o c}(\mathbb{R})$-valued functions and, consequently, the required estimate is an immediate corollary of estimates (1.1) and (1.5) (with $\phi=f, f_{u}^{\prime}, f_{u}^{\prime \prime}$, $t=t / \varepsilon$ and $T=\tau / \varepsilon$ ). Indeed, if $\tau \leq \varepsilon^{1 / 2}$, estimate (1.9) follows from (1.1) and, if 
$\varepsilon^{1 / 2} \leq \tau \leq 1$, then, due to estimate (1.5), we may estimate the right-hand side of (1.10) in terms of $\tau \alpha_{R}(\varepsilon / \tau) \leq \alpha_{R}\left(\varepsilon^{1 / 2}\right)$ and Lemma 1.1 is proven.

As usual, in order to study the attractors of non-autonomous equation (0.1), it is useful to consider not only the initial nonlinearity $f_{\varepsilon}$, but also all of the nonlinearities belonging to the hull of the initial nonlinearity $f_{\varepsilon}$.

Definition 1.2. Let $f(\varepsilon, t, z)$ satisfy the above assumptions and the function $f_{\varepsilon}$ be defined by (1.6). Then the hull $\mathcal{H}\left(f_{\varepsilon}\right)$ of $f_{\varepsilon}$ is the following set:

$$
\mathcal{H}\left(f_{\varepsilon}\right):=\left[T_{h} f_{\varepsilon}, h \in \mathbb{R}\right]_{C_{b}\left(\mathbb{R}, C_{l o c}^{3}(\Omega)\right)}, \quad\left(T_{h} f_{\varepsilon}\right)(u, t):=f_{\varepsilon}(u, t+h)
$$

where $[\cdot]_{V}$ denotes the closure in the space $V$. Then, since the functions $f_{\varepsilon}(u, t)$, $\partial_{u} f_{\varepsilon}(u, t), \partial_{u}^{2} f_{\varepsilon}(u, t)$ and $\partial_{u}^{3} f_{\varepsilon}(u, t)$ are assumed to be almost-periodic as $C_{l o c}(\mathbb{R})$ valued functions, then hull (1.11) is a compact subset of $C_{b}\left(\mathbb{R}, C_{l o c}(\mathbb{R})^{3}\right)$ (due to the Bochner-Amerio criterium, see [24]).

Lemma 1.3. Let the above assumptions hold. Then every function $\phi_{\varepsilon}(u, t)=$ $\phi(\varepsilon, u, z), z=t / \varepsilon$, belonging to the hull $\mathcal{H}\left(f_{\varepsilon}\right)$ of the initial nonlinearity $f_{\varepsilon}$ satisfies inequalities (1.1), (1.2) and (1.9) with the same constants $C_{R}$ and monotonic functions $Q$ and $\alpha_{R}$ as the initial nonlinearity $f_{\varepsilon}$.

Indeed, this assertion is a standard corollary of the definition of the hull (1.11), see, e.g. [8].

We now define the scale $E^{s}(\Omega), s \in \mathbb{R}$, of energy spaces associated with the hyperbolic equation (0.1) with Newmann boundary conditions via

$$
E^{s}(\Omega):=H_{N}^{s+1}(\Omega) \times H_{N}^{s}(\Omega), \quad \text { where } \xi_{u}(t):=\left(u(t), \partial_{t} u(t)\right) \in E^{s}(\Omega)
$$

and $H_{N}^{l}(\Omega):=D\left(\left(-\Delta_{x}+\lambda_{0}\right)_{N}^{l / 2}\right)$ is a scale of Hilbert spaces generated by the Laplace operator in $\Omega$ with Newmann boundary conditions. Then, as is well known,

$$
H_{N}^{l}(\Omega)= \begin{cases}H^{l}(\Omega), & -1 / 2<l<3 / 2, \\ H^{l}(\Omega) \cup\left\{\left.\partial_{n} u\right|_{\partial \Omega}=0\right\}, & 3 / 2<l<5 / 2\end{cases}
$$

where $H^{s}(\Omega)$ are the classical Sobolev spaces in $\Omega$, see [31] for the details. To simplify the notations, we will write below $E(\Omega)$ instead of $E^{0}(\Omega)$.

In the next two Lemmata, we obtain the analogues of estimate (1.9) for the case where the parameter $u$ depends on $t$ and $x(u=u(t, x))$.

Lemma 1.4. Let the above assumptions hold and let $u(t)=u(t, x)$ be a function satisfying

$$
\left\|\xi_{u}(t)\right\|_{E^{1}(\Omega)} \leq R, \forall t \in[0, T] .
$$

Then, for every $\varepsilon>0$ and $\phi_{\varepsilon} \in \mathcal{H}\left(f_{\varepsilon}\right)$, the following estimate holds:

$$
\left\|\int_{t}^{t+\tau}\left[\phi_{\varepsilon}(u(t), t)-\bar{f}(u(t))\right] d t\right\|_{L^{2}(\Omega)} \leq C_{R} \alpha_{C_{R}^{\prime}}(\varepsilon),
$$


for all $t, t+\tau \in[0, T]$ and $\tau \in[0,1]$ where the monotonic function $\alpha_{R}(\varepsilon)$ is the same as in (1.9) and the constants $C_{R}$ and $C_{R}^{\prime}$ are independent on $\varepsilon, t, T, \phi_{\varepsilon}$ and $u$.

Moreover, if $v(t)$ is another function such that $\xi_{v} \in L^{\infty}\left([0, T], E^{1}(\Omega)\right)$, then

$$
\begin{gathered}
\left\|\int_{t}^{t+\tau}\left[\partial_{u} \phi_{\varepsilon}(u(t), t)-\partial_{u} \bar{f}(u(t))\right] v(t) d t\right\|_{L^{2}(\Omega)} \leq \\
C_{R}\left\|\xi_{v}\right\|_{L^{\infty}\left([0, T], E^{1}(\Omega)\right)} \alpha_{C_{R}^{\prime}}(\varepsilon),
\end{gathered}
$$

for all $t, t+\tau \in[0, T]$ and $\tau \in[0,1]$.

Proof. We consider below only the case $\phi_{\varepsilon}=f_{\varepsilon}$ (the general case reduces to this one, due to Lemma 1.3). Let us first prove estimate (1.15). To this end, we use the following obvious identity:

$$
\begin{aligned}
& \frac{d}{d s} \int_{t}^{s}\left[f_{\varepsilon}(u(s), \kappa)-\bar{f}(u(s))\right] d \kappa=\left[f_{\varepsilon}(u(s), s)-\bar{f}(u(s))\right]+ \\
&+\partial_{t} u(s) \int_{t}^{s}\left[\partial_{u} f_{\varepsilon}(u(s), \kappa)-\partial_{u} \bar{f}(u(s))\right] d \kappa .
\end{aligned}
$$

Integrating this identity over $s \in[t, t+\tau]$, we derive

$$
\begin{aligned}
\int_{t}^{t+\tau}\left[f_{\varepsilon}(u(s), s)\right. & -\bar{f}(u(s))] d s=\int_{t}^{t+\tau}\left[f_{\varepsilon}(u(t+\tau), \kappa)-\bar{f}(u(t+\tau))\right] d \kappa- \\
& -\int_{t}^{t+\tau} \partial_{t} u(s)\left(\int_{t}^{s}\left[\partial_{u} f_{\varepsilon}(u(s), \kappa)-\partial_{u} \bar{f}(u(s))\right] d \kappa\right) d s
\end{aligned}
$$

We now note that estimate (1.14) and the embedding $H^{2}(\Omega) \subset C(\Omega)$ (we recall that $n=3$ ) imply that

$$
\|u(s)\|_{C(\Omega)}+\left\|\partial_{t} u(s)\right\|_{L^{2}(\Omega)} \leq C_{R}
$$

and, consequently, we may use inequality (1.9) (with $\phi_{\varepsilon}=f_{\varepsilon}$ and $\phi_{\varepsilon}=\partial_{u} f_{\varepsilon}$ ) in order to estimate the first term and the internal integral in the second term in the right-hand side of (1.18) which gives estimate (1.15).

Let us now consider estimate (1.16). Indeed, integrating by parts the left-hand side of (1.16), we have

$$
\begin{aligned}
\int_{t}^{t+\tau}\left[\partial_{u} f_{\varepsilon}(u(s), s)-\partial_{u} \bar{f}(u(s))\right] v(s) d s= \\
=v(t+\tau) \int_{t}^{t+\tau}\left[\partial_{u} f_{\varepsilon}(u(s), s)-\partial_{u} \bar{f}(u(s))\right] d s- \\
\quad-\int_{t}^{t+\tau} \partial_{t} v(s)\left(\int_{t}^{s}\left[\partial_{u} f_{\varepsilon}(u(\kappa), \kappa)-\partial_{u} \bar{f}(u(\kappa))\right] d \kappa\right) d s
\end{aligned}
$$

Estimating the first term and the internal integral in the second term in the righthand side of (1.20) using (1.18) (with $f_{\varepsilon}$ replaced by $\partial_{u} f_{\varepsilon}$ ) and Lemma 1.1, we derive estimate (1.16) and finish the proof of Lemma 1.4.

We now consider the analogue of estimate (1.15) for the case of less regular functions $u$. To this end, we need the following additional assumption on the growth of the nonlinearity $f$ :

$$
\left|f_{\varepsilon}(w, t)\right| \leq C\left(1+|w|^{3-\delta}\right), \quad\left|\partial_{u} f_{\varepsilon}(w, t)\right| \leq C(1+|w|), \quad \forall w \in \mathbb{R},
$$

where $C$ is independent of $t \in \mathbb{R}$ and $\delta$ is some positive number. 
Lemma 1.5. Let the nonlinearity $f_{\varepsilon}$ satisfy the additional assumption (1.21) and let $u(t, x)$ be a function satisfying

$$
\left\|\xi_{u}\right\|_{L^{\infty}([0, T], E(\Omega))} \leq R .
$$

Then, for every $\phi_{\varepsilon} \in \mathcal{H}\left(f_{\varepsilon}\right)$, estimate (1.15) holds (with some new monotonic function $\alpha_{R}$ which is independent of $t, \tau, \phi_{\varepsilon}$ and $u$ and tends to zero as $\varepsilon \rightarrow 0$ ).

Proof. As in Lemma 1.4, we give the proof for $\phi_{\varepsilon}=f_{\varepsilon}$ only and the general case is completely analogous due to Lemma 1.3. We also note, that in contrast to the proof of Lemma 1.4, we do not have now the estimate of the $C$-norm of the function $u$ and, consequently, we cannot directly apply Lemma 1.1 for estimating the integrals in the right-hand side of (1.18). In order to overcome this difficulty, we introduce, for every $N>0$ and $t \in[0, T]$, the following sets

$$
V_{t}^{N}(u):=\{x \in \Omega,|u(t, x)| \leq N\}, \quad W_{t}^{N}(u):=\Omega \backslash V_{t}^{N}(u) .
$$

Then, due to embedding $H^{1}(\Omega) \subset L^{6}(\Omega)$, growth restriction (1.21), estimate (1.22) and Hölder inequality, we have

$$
\left\|f_{\varepsilon}(u(t), s)\right\|_{L^{2}\left(W_{t}^{N}(u)\right)}+\|\bar{f}(u(s))\|_{L^{2}\left(W_{t}^{N}(u)\right)} \leq C_{R}\left|W_{t}^{N}(u)\right|^{\alpha_{1}} \leq C_{R}^{\prime} N^{-\alpha_{2}}
$$

where the positive constants $C_{R}, C_{R}^{\prime}, \alpha_{1}$ and $\alpha_{2}$ are independent of $N, \varepsilon, u, s$ and $t$. Thus, due to estimate $(1,24)$ and Lemma 1.1, we have

$$
\left\|\int_{t}^{t+\tau}\left[f_{\varepsilon}(u(\kappa), s)-\bar{f}(u(\kappa))\right] d s\right\|_{L^{2}(\Omega)} \leq
$$

$\left\|\int_{t}^{t+\tau}\left[f_{\varepsilon}(u(\kappa), s)-\bar{f}(u(\kappa))\right] d s\right\|_{L^{2}\left(V_{\kappa}^{N}(u)\right)}+C_{R}^{\prime} N^{-\alpha_{2}} \leq|\Omega|^{1 / 2} \alpha_{N}(\varepsilon)+C_{R}^{\prime} N^{-\alpha_{2}}$

where the constants $C_{R}^{\prime}$ and $\alpha_{2}$ are defined in (1.24) and the function $\alpha_{N}(\varepsilon)$ is the same as in Lemma 1.1. Fixing now the parameter $N=N(\varepsilon)$ in the right-hand side of (1.25) in an optimal way (i.e., as a solution of $N^{-\alpha_{1}}=\alpha_{N}(\varepsilon)$ ), we derive that the right-hand side of (1.25) tends to zero as $\varepsilon \rightarrow 0$ uniformly with respect to $u$, $t, \kappa \in[0, T]$ and $\tau \in[0,1]$. Moreover, arguing analogously, we can verify that (1.25) remains true with $f_{\varepsilon}$ and $\bar{f}$ replaced by $\partial_{u} f_{\varepsilon}$ and $\partial_{u} \bar{f}$ respectively. Inserting now these estimates into the right-hand side of (1.18) and using that $\left\|\partial_{t} u(s)\right\|_{L^{2}(\Omega)}^{2} \leq R$, we derive that

$$
\left\|\int_{t}^{t+\tau}\left[f_{\varepsilon}(u(s), s)-\bar{f}(u(s))\right] d s\right\|_{L^{1}(\Omega)} \leq \alpha_{R}^{\prime}(\varepsilon)
$$

where the monotonic function $\alpha_{R}^{\prime}(\varepsilon)$ is independent of $u, t$ and $\tau \in[0,1]$ and tends to zero as $\varepsilon \rightarrow 0$. In order to deduce the analogue of (1.26) for the $L^{2}$-norm, there remains to note that, due to estimate (1.22), embedding $H^{1}(\Omega)$ and growth restrictions (1.21), we have

$$
\left\|\int_{t}^{t+\tau}\left[f_{\varepsilon}(u(s), s)-\bar{f}(u(s))\right] d s\right\|_{L^{2+\delta_{1}(\Omega)}} \leq C_{R}^{\prime \prime}
$$


for some positive exponent $\delta_{1}=\delta_{1}(\delta)$. Estimates (1.26) and (1.27) together with the interpolation inequality give estimate (1.15) and finish the proof of Lemma 1.5.

To conclude the section, we discuss the rate of convergence to zero of the functions $\alpha_{R}(\varepsilon)$ in Lemma 1.1 as $\varepsilon \rightarrow 0$. To this end, we assume, in addition, that the function $f(0, u, z)-\bar{f}(u)$ has a bounded primitive $F(u, z)$, i.e.

$$
\partial_{z} F(u, z)=f(0, u, z)-\bar{f}(u)
$$

and

$$
|F(u, z)|+\left|\partial_{u} F(u, z)\right|+\left|\partial_{u}^{2} F(u, z)\right| \leq Q(|u|),
$$

for some monotonic function $Q$ which is independent of $z$.

Under these assumptions, we have the linear rate of decaying of $\alpha_{R}$ as $\varepsilon \rightarrow 0$.

Lemma 1.6. Let the additional assumptions (1.28) and (1.29) hold. Then the function $\alpha_{R}(\varepsilon)$ introduced in Lemma 1.1 (and used in the right-hand sides of estimates (1.15) and (1.16)) possesses the following upper bound:

$$
\alpha_{R}(\varepsilon) \leq C_{R} \varepsilon
$$

where the constant $C_{R}$ is independent of $\varepsilon$.

Proof. Indeed, due to estimate (1.2) it is sufficient to estimate the term in the lefthand side of (1.10) and its first and second derivatives with respect to $u$. In order to do so, we transform the right-hand side of (1.10), using (1.28), as follows:

$$
\int_{t}^{t+\tau}[f(0, u, t / \varepsilon)-\bar{f}(u)] d t=\varepsilon(F(u, t / \varepsilon+\tau / \varepsilon)-F(u, t / \varepsilon)) .
$$

Estimate (1.30) is now an immediate corollary of assumption (1.29). Lemma 1.6 is proven.

Remark 1.7. By definition, see (1.7), the function $f_{\varepsilon}(0, u, z)-\bar{f}(u)$ has zero mean. Therefore, condition (1.29) will be always satisfied if the function $f(0, u, z)$ is periodic with respect to $z$. Consequently, (1.30) is automatically satisfied for periodic nonlinearities $f$. Unfortunately, for more general quasi-periodic or almostperiodic functions, the sole zero mean assumption is not sufficient in order to obtain the bounded primitive (since the so-called small denominators may appear under the integration, see [23] or [24]). Thus, in this case, some additional assumptions (e.g., some decay assumptions on the Fourier amplitudes of $f(0, u, z)$ or some kind of Diophantine conditions on its Fourier frequences) are required in order to have estimate (1.30), see [14], [16], [17] and [24].

$\S 2$ The LOCAL AVERAGing AND The GLOBAL EXISTENCE OF STRONG SOLUTIONS.

In this section, we prove the existence of a global strong solution of the nonaveraged equation (0.1) if $\varepsilon$ is small enough and the initial $E^{1}$-energy is not very large. We obtain this result by comparison of the solution of equation (0.1) with the corresponding solution of the averaged equation which obviously has the following form:

$$
\left\{\begin{array}{l}
\partial_{t}^{2} \bar{u}+\gamma \partial_{t} \bar{u}-\Delta_{x} \bar{u}+\lambda_{0} \bar{u}+\bar{f}(\bar{u})=g \\
\left.\xi_{\bar{u}}\right|_{t=\tau}=\xi^{\tau},\left.\quad \partial_{n} \bar{u}\right|_{\partial \Omega}=0
\end{array}\right.
$$

We start by recalling the classical result on the global solvability of equation (2.1). 
Theorem 2.1. Let the function $\bar{f}$ satisfy assumptions (1.8) and $g \in L^{2}(\Omega)$. Then, for every $\tau \in \mathbb{R}$ and $\xi^{\tau} \in E^{1}(\Omega)$, equation (2.1) has a unique solution $\bar{u}(t)$ which satisfies the following estimate:

$$
\left\|\xi_{\bar{u}}(t)\right\|_{E^{1}(\Omega)} \leq Q\left(\left\|\xi_{\bar{u}}(\tau)\right\|_{E^{1}(\Omega)}\right) e^{-\alpha(t-\tau)}+Q\left(\|g\|_{L^{2}(\Omega)}\right)
$$

where $t \geq \tau$ and the positive constant $\alpha$ and the monotonic function $Q$ are independent of $\tau, t, \xi^{\tau}$ and $g$.

The proof of this result can be found, e.g. in [3].

Let us now consider the non-averaged equation (0.1). As usual, in order to study the long-time behavior of solutions of this equation, it is useful to consider a family of equations of the form (0.1) with all nonlinearities $\phi_{\varepsilon}$, belonging to the hull $\mathcal{H}\left(f_{\varepsilon}\right)$ of the initial nonlinearity. To be more precise, for every $\varepsilon>0, \tau \in \mathbb{R}$ and every $\phi_{\varepsilon} \in \mathcal{H}\left(f_{\varepsilon}\right)$, we consider the following problem:

$$
\left\{\begin{array}{l}
\partial_{t}^{2} u+\gamma \partial_{t} u-\Delta_{x} u+\lambda_{0} u+\phi_{\varepsilon}(u, t)=g, \\
\left.\xi_{u}\right|_{t=\tau}=\xi^{\tau},\left.\quad \partial_{n} u\right|_{\partial \Omega}=0 .
\end{array}\right.
$$

We first establish the existence of a solution $u(t)$ on a finite interval $[0, T]$ if $\varepsilon$ is small enough.

Theorem 2.2. Let the function $f(\varepsilon, u, z)$ satisfy the assumptions of Lemma 1.1. Then, for every $T>0$ and $R>0$, there exists $\varepsilon_{0}=\varepsilon_{0}(T, R)$ such that for every $\varepsilon<\varepsilon_{0}, \phi_{\varepsilon} \in \mathcal{H}\left(f_{\varepsilon}\right), \tau \in \mathbb{R}$ and $\xi^{\tau} \in E^{1}(\Omega)$ which satisfies $\left\|\xi^{\tau}\right\|_{E^{1}(\Omega)} \leq R$, equation (2.3) has a unique strong solution $u_{\varepsilon}(t)$ on the interval $t \in[\tau, \tau+T]$ and the following estimate holds:

$$
\left\|\xi_{u_{\varepsilon}}(t)-\xi_{\bar{u}}(t)\right\|_{E^{1}(\Omega)} \leq C_{T, R} \alpha_{C_{R}}(\varepsilon)^{\beta}, \forall t \in[\tau, \tau+T]
$$

where the function $\alpha_{R}(\varepsilon)$ is the same as in Lemma 1.1 and positive constants $C_{T, R}$, $C_{R}$ and $\beta$ are independent of $\tau, \xi^{\tau}, \varepsilon \leq \varepsilon_{0}$ and $\phi_{\varepsilon} \in \mathcal{H}\left(f_{\varepsilon}\right)$.

Proof. We are going to construct the desired solution $u_{\varepsilon}(t)$ as a small perturbation of the corresponding solution $\bar{u}(t)$ of the averaged equation (2.1) (with the same initial conditions) based on estimates of Lemma 1.4, estimate (2.2) and the implicit function theorem. For simplicity, we below consider only the case $\phi_{\varepsilon}=f_{\varepsilon}$ and $\tau=0$ (the general case is completely analogous to this one, thanks to Lemma 1.3). We introduce a new unknown function $w_{\varepsilon}(t):=u_{\varepsilon}(t)-\bar{u}(t)$ which should satisfy the following equation:

$$
\begin{aligned}
\partial_{t}^{2} w_{\varepsilon}+\gamma \partial_{t} w_{\varepsilon}-\Delta_{x} w_{\varepsilon}+\lambda_{0} w_{\varepsilon} & +\left[\bar{f}\left(w_{\varepsilon}+\bar{u}(t)\right)-\bar{f}(\bar{u}(t))\right]= \\
= & {\left[f_{\varepsilon}\left(w_{\varepsilon}+\bar{u}, t\right)-\bar{f}\left(w_{\varepsilon}+\bar{u}\right)\right],\left.\quad \xi_{w_{\varepsilon}}\right|_{t=0}=0 . }
\end{aligned}
$$

We are going to apply the implicit function theorem to equation (2.5). To this end, we define the space

$$
\mathcal{L}_{T}:=\left\{\xi_{u} \in C\left([0, T], E^{1}(\Omega)\right), \xi_{u}(0)=0\right\}
$$

and invert the linear part of equation (2.5). Then, we obtain the relation

$$
\xi_{w_{\varepsilon}}+\mathbb{H}_{T}\left(\bar{f}\left(w_{\varepsilon}+\bar{u}\right)-\bar{f}(\bar{u})\right) \underset{10}{=\mathbb{H}_{T}}\left(f_{\varepsilon}\left(w_{\varepsilon}+\bar{u}, t\right)-\bar{f}\left(w_{\varepsilon}+\bar{u}\right)\right)
$$


where $\mathbb{H}_{T}: h \rightarrow \xi_{\theta}$ is the solving operator of the linear hyperbolic problem

$$
\partial_{t}^{2} \theta+\gamma \partial_{t} \theta-\Delta_{x} \theta+\lambda_{0} \theta=h, t \in[0, T], \xi_{\theta}(0)=0
$$

Let us introduce an operator $\Phi: \mathcal{L}_{T} \times E^{1}(\Omega) \times \mathbb{R}_{+} \rightarrow \mathcal{L}_{T}$ as follows:

$$
\Phi\left(\xi_{w}, \xi^{0}, \varepsilon\right):=\xi_{w}-\mathbb{T}_{0}\left(\xi_{w}, \xi^{0}\right)+\mathbb{T}_{1}\left(\xi_{w}, \xi^{0}, \varepsilon\right)
$$

where

$\mathbb{T}_{0}(\cdot):=\mathbb{H}_{T}\left(\bar{f}\left(w+\bar{u}\left(\xi^{0}\right)\right)-\bar{f}\left(\bar{u}\left(\xi^{0}\right)\right)\right), \mathbb{T}_{1}(\cdot)=\mathbb{H}_{T}\left(f_{\varepsilon}\left(w+\bar{u}\left(\xi^{0}\right), t\right)-\bar{f}\left(w+\bar{u}\left(\xi^{0}\right)\right)\right)$

and $\bar{u}\left(\xi^{0}\right)$ is a solution of $(2.1)$ on $[0, T]$ with $\xi_{\bar{u}}(0)=\xi^{0}$. In order to study function (2.9), we need the following lemma

Lemma 2.3. The operator $\mathbb{T}_{1}$ satisfies the following estimate:

$$
\left\|\mathbb{T}_{1}\left(\xi_{w}, \xi^{0}, \varepsilon\right)\right\|_{\mathcal{L}_{T}}+\left\|D_{\xi_{w}} \mathbb{T}_{1}\left(\xi_{w}, \xi^{0}, \varepsilon\right)\right\|_{\mathcal{L}\left(\mathcal{L}_{T}, \mathcal{L}_{T}\right)} \leq C_{T, R} \alpha_{C_{R}}(\varepsilon)^{\beta}
$$

if $\left\|\xi_{w}\right\|_{\mathcal{L}_{T}}+\left\|\xi^{0}\right\|_{E^{1}(\Omega)} \leq 2 R$, where the function $\alpha_{R}(\varepsilon)$ is the same as in Lemma 1.1 and the positive constants $C_{T, R}, C_{R}$ and $\beta$ are independent of $\varepsilon, \xi^{0}$ and $\xi_{w}$.

Proof. Indeed, thanks to estimates (1.15), (2.2) and (A.4) (with $s=0$ ), we have

$$
\left\|\mathbb{T}_{1}\left(\xi_{w}, \xi^{0}, \varepsilon\right)\right\|_{C\left([0, T], E^{-1}(\Omega)\right)} \leq C_{T, R}^{\prime} \alpha_{C_{R}^{\prime}}(\varepsilon) .
$$

On the other hand, it follows from assumption (1.1) and the dissipative estimate (2.2) that $\left\|f_{\varepsilon}(w(t)+\bar{u}(t), t)-\bar{f}(w(t)+\bar{u}(t))\right\|_{H^{2}(\Omega)} \leq C_{R}^{\prime \prime}$ and, consequently, due to (A.2), we have

$$
\left\|\mathbb{T}_{1}\left(\xi_{w}, \xi^{0}, \varepsilon\right)\right\|_{C\left([0, T], E^{1+\delta}(\Omega)\right)} \leq C_{R}^{\prime}
$$

for some $0<\delta<1 / 2$. Inequalities (2.11) and (2.12) together with the interpolation inequality give (2.10). Estimate of the derivative can be proven analogously, only one should use estimate (1.16) instead of (1.15). Lemma 2.3 is proven.

Estimate (2.10) implies, in particular, that

$$
\Phi\left(0, \xi^{0}, 0\right) \equiv 0
$$

Moreover, since the differentiability of the operator $\mathbb{T}_{0}$ with respect to $\xi_{w}$ is obvious, then

$$
D_{\xi_{w}} \Phi\left(0, \xi^{0}, 0\right) \xi_{\theta}=\xi_{\theta}+\mathbb{H}_{T}\left(\bar{f}^{\prime}(\bar{u}(\xi)) \theta\right)
$$

and $\Phi\left(\xi_{w}, \xi^{0}, \varepsilon\right)$ and $D_{\xi_{w}} \Phi\left(\xi_{w}, \xi^{0}, \varepsilon\right)$ tend to $\Phi\left(\xi_{w}, \xi^{0}, 0\right)$ and $D_{\xi_{w}} \Phi\left(\xi_{w}, \xi^{0}, 0\right)$ as $\varepsilon \rightarrow 0$ respectively (and this convergence is uniform with respect to $\xi^{0}$ ). Thus, in order to deduce estimate (2.4) from (2.7) and the implicit function theorem, there only remains to verify that the operator $(2.14)$ is (uniformly with respect to $\xi^{0}$ ) invertible in $\mathcal{L}_{T}$. 
Lemma 2.4. The equation

$$
D_{\xi_{w}} \Phi\left(0, \xi^{0}, 0\right) \xi_{\theta}=\xi_{v}
$$

has a unique solution $\xi_{\theta}$, for every $\xi_{v} \in \mathcal{L}_{T}$, and the following estimate holds:

$$
\left\|\xi_{\theta}\right\|_{\mathcal{L}_{T}} \leq C_{R, T}\left\|\xi_{v}\right\|_{\mathcal{L}_{T}}
$$

for all $\xi^{0} \in E^{1}(\Omega)$ such that $\left\|\xi^{0}\right\|_{E^{1}(\Omega)} \leq R$.

Proof. Indeed, let $w(t):=\theta(t)-v(t)$, where $\xi_{\theta}$ is a solution of (2.15). Then, this function obviously satisfies the equation:

$$
\partial_{t}^{2} w+\gamma \partial_{t} w-\Delta_{x} w+\lambda_{0} w+f^{\prime}(\bar{u}(t))(w(t)+v(t))=0, \quad \xi_{w}(0)=0 .
$$

We recall that, due to estimate (2.2), we have

$$
\left\|f^{\prime}(\bar{u}(t))\right\|_{C(\Omega)}+\left\|f^{\prime}(\bar{u}(t))\right\|_{H^{2}(\Omega)} \leq C_{R}, \quad \forall t \geq 0
$$

The existence of a solution of (2.17) and estimate (2.16) is now an immediate corollary of this estimate and the classical $E^{1}$-energy estimates for the solutions of linear hyperbolic equations, see e.g., [3] or [29]. Lemma 2.4 is proven.

Thus, we have verified that operator (2.9) (in which $\xi^{0}$ is interpreted as a parameter) satisfies all of the assumptions of the implicit function theorem and, consequently, the desired solution $\xi_{w_{\varepsilon}}$ can be found in a unique way from the equation

$$
\Phi\left(\xi_{w_{\varepsilon}}, \xi^{0}, \varepsilon\right) \equiv 0
$$

if $\varepsilon$ is small enough. Moreover, estimate (2.4) is now a standard corollary of estimates (2.10). Theorem 2.2 is proven.

Remark 2.5. We note that the right-hand side of (2.4) tends to zero as $\varepsilon \rightarrow 0$ and, consequently, the solution $u_{\varepsilon}(t)$ of equation (2.3) with rapidly oscillating in time coefficients tends to the corresponding solution $\bar{u}(t)$ as $\varepsilon \rightarrow 0$ on every finite interval $[\tau, \tau+T]$. Thus, Theorem 2.2 can be interpreted as the analogue of the first Bogolubov's theorem for the hyperbolic equation of the form (2.3), see [5], [26].

We now recall that, only the averaged nonlinearity $\bar{f}$ is assumed to satisfy the dissipativity and growth assumptions which guarantee the existence of the global solutions. Thus, in general, we do not have the existence of a global solution for equation (2.3) if $\varepsilon>0$. Nevertheless, estimates (2.2) and (2.4) allow to prove the global existence if $\varepsilon>0$ is small enough and the $E^{1}$-energy of the initial data $\xi^{\tau}$ is not very large. To be more precise, the following result holds.

Corollary 2.6. Let the assumptions of Theorem 2.2 hold. Then, there exists $\varepsilon_{0}>0$ and a monotone decreasing function $R_{0}:\left(0, \varepsilon_{0}\right] \rightarrow \mathbb{R}_{+}$such that $\lim _{\varepsilon \rightarrow 0} R_{0}(\varepsilon)=\infty$ and, for every $\varepsilon \leq \varepsilon_{0}, \tau \in \mathbb{R}, \phi_{\varepsilon} \in \mathcal{H}\left(f_{\varepsilon}\right)$ and $\xi^{\tau} \in E^{1}(\Omega)$ which satisfies the condition

$$
\left\|\xi^{\tau}\right\|_{E^{1}(\Omega)} \leq R_{0}(\varepsilon)
$$


equation (2.3) possesses a unique global solution $u \in L^{\infty}\left(\mathbb{R}_{+}, E^{1}(\Omega)\right)$ and the following estimate is valid:

$$
\left\|\xi_{u}(t)\right\|_{E^{1}(\Omega)} \leq \tilde{Q}\left(\left\|\xi_{u}(\tau)\right\|_{E^{1}(\Omega)}\right) e^{-\alpha(t-\tau)}+\tilde{Q}\left(\|g\|_{L^{2}(\Omega)}\right)
$$

where positive constant $\alpha$ and monotonic function $\tilde{Q}$ are independent of $\varepsilon, \tau, \phi_{\varepsilon}$ and $\xi^{\tau}$.

Proof. Instead of constructing the function $R_{0}(\varepsilon)$, it is more convenient to construct the inverse function $\varepsilon_{0}(R)$. Indeed, let $R$ be an arbitrary sufficiently large number $\left(R \geq 4 Q\left(\|g\|_{L^{2}(\Omega)}\right)\right.$ where $Q$ is the same as in estimate $\left.(2.2)\right)$. Then, we fix $T=$ $T(R)$ as a solution of the following equation

$$
Q(R) e^{-\alpha T}=Q\left(\|g\|_{L^{2}(\Omega)}\right)
$$

Finally, we fix $\varepsilon_{0}=\varepsilon_{0}(R)$ such that

$$
C_{T, R} \alpha_{C_{R}}\left(\varepsilon_{0}\right)^{\beta}=Q\left(\|g\|_{L^{2}(\Omega)}\right)
$$

where the constants $C_{R}, \beta$ and $C_{T, R}$ and the function $\alpha_{R}(\varepsilon)$ are the same as in Theorem 2.2. We claim that, if $\varepsilon \leq \varepsilon_{0}$ and $\left\|\xi^{\tau}\right\|_{E^{1}(\Omega)} \leq R$, then equation (2.3) has a solution $u(t)$ which satisfies estimate (2.21). Indeed, thanks to Theorem 2.2, equation (2.3) has a solution $u(t)$ which satisfies the estimate

$$
\left\|\xi_{u}(t)-\xi_{\bar{u}}(t)\right\|_{E^{1}(\Omega)} \leq Q\left(\|g\|_{L^{2}(\Omega)}\right), \quad \forall t \in[\tau, \tau+T]
$$

where $\bar{u}(t)$ is the corresponding solution of the averaged equation (2.1). On the other hand, thanks to estimate (2.2) and equation (2.23), we have the estimate $\left\|\xi_{\bar{u}}(\tau+T)\right\|_{E^{1}(\Omega)} \leq 2 Q\left(\|g\|_{L^{2}(\Omega)}\right)$. Combining this estimate with (2.25), we derive that

$$
\left\|\xi_{u}(\tau+T)\right\|_{E^{1}(\Omega)} \leq 3 Q\left(\|g\|_{L^{2}(\Omega)}\right)<R .
$$

Thus, we may apply again Theorem 2.2 in order to construct the solution of equation (2.3) on the interval $[\tau+T, \tau+2 T]$ with $\left.\xi_{u}\right|_{t=\tau+T}=\xi_{u}(\tau+T)$. Iterating this procedure, we obtain the global solution $u(t)$ of equation (2.3) defined for every $t \in[\tau,+\infty)$ such that

$$
\left\|\xi_{u}(\tau+n T)\right\|_{E^{1}(\Omega)} \leq 3 Q\left(\|g\|_{L^{2}(\Omega)}\right), \quad \forall n \in \mathbb{N}
$$

Estimate (2.21) is now a corollary of (2.2), (2.27) and (2.25). Since the strong solution $\xi_{u} \in L^{\infty}\left([\tau,+\infty], E^{1}(\Omega)\right)$ is unique then Corollary 2.6 is proven.

Thus, for sufficiently small $\varepsilon$ and every $\phi_{\varepsilon} \in \mathcal{H}\left(f_{\varepsilon}\right)$, equation (2.3) defines a family of solving operators

$$
\begin{aligned}
& U_{\phi_{\varepsilon}}(t, \tau): B_{R_{0}(\varepsilon)} \rightarrow E^{1}(\Omega), \quad t, \tau \in \mathbb{R}, t \geq \tau \text { on the ball } \\
& B_{R_{0}(\varepsilon)}:=\left\{\xi \in E^{1}(\Omega),\|\xi\|_{E^{1}(\Omega)} \leq R_{0}(\varepsilon)\right\} \\
& 13
\end{aligned}
$$


via $U_{\phi_{\varepsilon}}(t, \tau) \xi^{\tau}=\xi_{u}(t)$, where $u(t)$ is a solution of (2.3) which exists thanks to Corollary 2.6. Moreover, these families, obviously, satisfy the following translation identity:

$$
U_{\phi_{\varepsilon}}(t+s, \tau+s)=U_{T_{s} \phi_{\varepsilon}}(t, \tau),
$$

for every $\phi_{\varepsilon} \in \mathcal{H}\left(f_{\varepsilon}\right), t, \tau, s \in \mathbb{R}$ and $t \geq \tau$, where the shift operator is defined in (1.11). We also note that the limit case $\varepsilon=0$ corresponds to the autonomous equation (2.1) whose solutions exist globally for every $\xi^{\tau} \in E^{1}(\Omega)$, consequently, this equation generates a semigroup $\left\{S_{t}, t \geq 0\right\}$ in the whole phase space $E^{1}(\Omega)$ :

$$
S_{t} \xi:=U_{\bar{f}}(t, 0) \xi, \quad \forall \xi \in E^{1}(\Omega), \quad \text { and } S_{t} \circ S_{h}=S_{t+h} \quad \forall t, h \in \mathbb{R}_{+} .
$$

The rest of this section is devoted to study the analytic properties of operators (2.28). We start with the standard result on the differentiability with respect to the initial data $\xi^{\tau}$.

Proposition 2.7. Let the assumptions of Corollary 2.6 hold. Then, for every $\xi^{\tau} \in$ $B_{R_{0}(\varepsilon)}$, the function $\xi^{\tau} \rightarrow U_{\phi_{\varepsilon}}(t, \tau) \xi^{\tau}$ is Frechet differentiable and its derivative $D_{\xi^{\tau}} U_{\phi_{\varepsilon}}(t, \tau)$ can be computed as follows: $\left[D_{\xi^{\tau}} U_{\phi_{\varepsilon}}(t, \tau) \xi^{\tau}\right] \theta:=\xi_{w_{\theta}}(t)$, where $\theta \in$ $E^{1}(\Omega)$ is an arbitrary vector and $w_{\theta}(t)$ is a solution of the following equation of variations:

$$
\left\{\begin{array}{l}
\partial_{t}^{2} w_{\theta}+\gamma \partial_{t} w_{\theta}-\Delta_{x} w_{\theta}+\lambda_{0} w_{\theta}+\partial_{u} \phi_{\varepsilon}(u(t), t) w_{\theta}=0, \\
\left.\xi_{w_{\theta}}\right|_{t=\tau}=\theta, \quad u(t):=U_{\phi_{\varepsilon}}(t, \tau) \xi^{\tau} .
\end{array}\right.
$$

Moreover, this derivative satisfies the following estimates:

$$
\left\|D_{\xi^{\tau}} U_{\phi_{\varepsilon}}(t, \tau)\right\|_{\mathcal{L}\left(E^{1}, E^{1}\right)} \leq C e^{K(t-\tau)}
$$

where the constants $C$ and $K$ depend on $\left\|\xi^{\tau}\right\|_{E^{1}}$, but are independent of $\varepsilon, t, \tau$ and $\phi_{\varepsilon}$, and, for every $\xi_{1}^{\tau}$ and $\xi_{2}^{\tau}$ belonging to $B_{R_{0}(\varepsilon)}$, we have

$$
\left\|D_{\xi^{\tau}} U_{\phi_{\varepsilon}}(t, \tau)\left(\xi_{1}^{\tau}\right)-D_{\xi^{\tau}} U_{\phi_{\varepsilon}}(t, \tau)\left(\xi_{2}^{\tau}\right)\right\|_{\mathcal{L}\left(E^{1}, E^{1}\right)} \leq C e^{K(t-\tau)}\left\|\xi_{1}^{\tau}-\xi_{2}^{\tau}\right\|_{E^{1}(\Omega)}
$$

where the constants $C$ and $K$ are also independent of $t, \tau, \varepsilon$ and $\phi_{\varepsilon}$.

Indeed, Proposition 2.7 is a standard corollary of estimate (A.2) with $s=1$, estimate (2.21) and assumption (1.1) on the nonlinearity $f$ (since all of these estimates are uniform with respect to $\varepsilon$, then (2.32) and (2.33) will be also uniform with respect to $\varepsilon$ ), so we left its rigorous proof to the reader.

We now establish the convergence of operators (2.28) to the limit semigroup $S_{t}$ as $\varepsilon \rightarrow 0$.

Proposition 2.8. Let the assumptions of Corollary 2.6 hold. Then the operators $U_{\phi_{\varepsilon}}(t, \tau)$ tend to $S_{t-\tau}$ as $\varepsilon \rightarrow 0$ in the following sense:

$$
\begin{aligned}
& \left\|U_{\phi_{\varepsilon}}(t, \tau)\left(\xi^{\tau}\right)-S_{t-\tau}\left(\xi^{\tau}\right)\right\|_{E^{1}(\Omega)}+ \\
& \quad+\left\|D_{\xi^{\tau}} U_{\phi_{\varepsilon}}(t, \tau)\left(\xi^{\tau}\right)-D_{\xi^{\tau}} S_{t-\tau}\left(\xi^{\tau}\right)\right\|_{\mathcal{L}\left(E^{1}, E^{1}\right)} \leq C_{R} e^{K(t-\tau)} \alpha_{C_{R}}(\varepsilon)^{\beta}
\end{aligned}
$$

where the constants $C_{R}$ and $K$ depend on $R$ (we recall that $\left\|\xi^{\tau}\right\|_{E^{1}(\Omega)} \leq R \leq R_{0}(\varepsilon)$ ) but are independent of $t, \tau, \varepsilon$ and $\phi_{\varepsilon}$ and the positive constant $\beta$ and the monotonic function $\alpha_{R}(\varepsilon)$ are the same as in Theorem 2.2. 
Proof. Estimate (2.34) can be easily deduced from the implicit function theorem (to this end, we only need to verify that function (2.9) is differentiable with respect to $\left.\xi^{0}\right)$, but we prefer to give an independent proof of this fact. We restrict ourselves to consider only the case $\phi_{\varepsilon}=f_{\varepsilon}$ and $\tau=0$ (the general case is analogous due to Lemma 1.3). Let us first verify estimate (2.34) for the first term in the left-hand side. Indeed, let $u_{\varepsilon}(t):=U_{f_{\varepsilon}}(t, 0) \xi^{0}$ and $\bar{u}(t):=S_{t} \xi^{0}$ be solutions of equations (2.3) and (2.1) respectively and let $w_{\varepsilon}(t):=u_{\varepsilon}(t)-\bar{u}(t)$. Then, this function satisfies equation (2.5). Let us now introduce a new function $\theta(t)$ as a solution of the following auxiliary equation:

$$
\partial_{t}^{2} \theta+\gamma \partial_{t} \theta-\Delta_{x} \theta+\lambda_{0} \theta=\left[f_{\varepsilon}\left(u_{\varepsilon}(t), t\right)-\bar{f}\left(u_{\varepsilon}(t)\right)\right]:=h_{u_{\varepsilon}}(t), \quad \xi_{\theta}(0)=0 .
$$

Estimates (1.1), (1.15) and (2.21) imply that

$$
\left\|\int_{t}^{t+\tau} h_{u_{\varepsilon}}(s) d s\right\|_{L^{2}(\Omega)} \leq C_{R} \alpha_{C_{R}}(\varepsilon) \text { and }\left\|h_{u_{\varepsilon}}(t)\right\|_{H^{2}(\Omega)} \leq C_{R}^{\prime}
$$

for every $t \in \mathbb{R}_{+}, \tau \in[0,1]$ where the constant $C_{R}$ depends only on $R$ and the function $\alpha_{R}$ is the same as in (1.15). Thus, thanks to estimates (A.2), (A.4) and the interpolation inequality, we have (see the proof of Lemma 2.3)

$$
\|\theta(t)\|_{E^{1}(\Omega)} \leq C_{R}^{\prime \prime} \alpha_{C_{R}}(\varepsilon)^{\beta}, \quad t \in \mathbb{R}_{+}
$$

where the constants $C_{R}^{\prime \prime}$ and $C_{R}$ depend only on $R$ and the positive constant $\beta$ and the function $\alpha_{R}$ are the same as in Theorem 2.1.

Let now $v_{\varepsilon}(t):=w_{\varepsilon}(t)-\theta(t)$. Then, this function satisfies

$$
\begin{aligned}
\partial_{t}^{2} v_{\varepsilon}+\gamma \partial_{t} v_{\varepsilon}- & \Delta_{x} v_{\varepsilon}+\lambda_{0} v_{\varepsilon}= \\
& =\left[\bar{f}(\bar{u}(t))-\bar{f}\left(\bar{u}(t)+v_{\varepsilon}(t)+\theta(t)\right)\right]:=h_{\theta}(t), \quad \xi_{v_{\varepsilon}}(0)=0 .
\end{aligned}
$$

Using now estimates $(1.1),(2.2),(2.21)$ and the fact that the space $H^{2}(\Omega)$ is an algebra (we recall that $n=3$ ), we deduce in a standard way that

$$
\left\|h_{\theta}(t)\right\|_{H^{1}(\Omega)} \leq C_{R}^{\prime \prime \prime}\left(\left\|v_{\varepsilon}(t)\right\|_{H^{2}(\Omega)}+\|\theta(t)\|_{H^{2}(\Omega)}\right)
$$

where $C_{R}^{\prime \prime \prime}$ depends only on $R$. Applying estimate (A.2) to equation (2.38) and using estimates (2.37), (2.39) and the Gronwall's inequality, we finally derive that

$$
\left\|v_{\varepsilon}(t)\right\|_{E^{1}(\Omega)} \leq C_{R} e^{K t} \alpha_{C_{R}}(\varepsilon)^{\beta} .
$$

Thus, the first term in the right-hand side of (2.34) is estimated. The second term can be estimated analogously: we should consider the difference between the nonaveraged (cf. (2.31)) and averaged equation of variations and use estimate (2.16) instead of (2.15). Proposition 2.8 is proven.

Remark 2.9. We now discuss some generalizations of the obtained results. We first note that the almost-periodicity of functions $f_{\varepsilon}$ with respect to $t$ is necessary only for the proof of estimate (1.9) in Lemma 1.1. Thus, all the results of this section remain true if, instead of the almost-periodicity, we postulate the existence of a function $\bar{f}(u)$ which satisfies estimates (1.9). 
We also note that, although we consider only the spatially homogeneous nonlinearities $f_{\varepsilon}(u, t)$, this assumption is not crucial for our method and the results remain true for more general nonlinearities $f_{\varepsilon}(u, t, x)$ (under some smoothness assumptions on $f_{\varepsilon}$ with respect to $x$ ).

To conclude, we observe that the Newmann boundary condition is also not essential for our technique. The only difference (e.g., with the case of Dirichlet boundary conditions) is that the analogue of estimate (A.2) with $s>1 / 2$ requires the boundary condition $\left.h(t)\right|_{\partial \Omega}=0$ (in fact, we do not know whether or not estimate (A.2) holds without this assumption). Thus, in the case of Dirichlet boundary conditions, we need to assume, in addition, that

$$
f_{\varepsilon}(0, t) \equiv 0, \quad \forall t \in \mathbb{R} .
$$

\section{$\S 3$ The attractors and their averaging.}

In this section, we start to study the long-time behavior of solutions of (2.3) in the case where $\varepsilon>0$ is small enough. We recall that, in contrast to the case $\varepsilon=0$, for positive $\varepsilon$, we have the global existence of a solution for the initial data belonging to the ball $B_{R_{0}(\varepsilon)}$ of radius $R_{0}(\varepsilon)$ in $E^{1}(\Omega)$ only (see Sections 6 and 7 for the discussion of the case where the initial data does not belong to this ball). So, it is natural to consider this ball as the phase space for problem (2.3) and, thus, to construct the attractors for the solutions whose initial data belong to $B_{R_{0}(\varepsilon)}$ only. We also note that the main estimate $(2.21)$ is not strong enough to conclude that the solving operators (2.28) map $B_{R_{0}(\varepsilon)}$ to itself, for every $t \geq \tau$. Nevertheless, it follows from (2.21) that there exists $T_{0}=T_{0}(\varepsilon)$ which is independent of $t, \tau$ and $\phi_{\varepsilon}$ such that

$$
U_{\phi_{\varepsilon}}(t, \tau): B_{R_{0}(\varepsilon)} \rightarrow B_{R_{0}(\varepsilon)}, \text { for all } t, \tau \in \mathbb{R}, \quad t-\tau \geq T_{0} \text { and } \phi_{\varepsilon} \in \mathcal{H}\left(f_{\varepsilon}\right)
$$

which is quite enough for the attractors theory.

It is worth to recall here that, in contrast to the limit equation (2.1), equations (2.3) are non-autonomous, thus, operators (3.1) do not generate a semigroup in the phase space $B_{R_{0}(\varepsilon)}$ and the standard concept of a global attractor is not directly applicable here. Up to now, two major possibilities to generalize the concept of a global attractor to non-autonomous equations are known. The first one is to reduce the non-autonomous dynamical system to the autonomous one defined on the properly extended phase space. This approach naturally leads to the concept of the so-called uniform attractor, see [8], [19] and the explanations below. The alternative approach is the so-called pullback attractor which treats the attractor of the non-autonomous equation as a non-autonomous set as well and, therefore, does not require the reducing to the autonomous system, see [12] and [22].

We start our exposition by the uniform attractor (and the pullback attractor will be discussed at the end of the section). To this end (following the standard scheme, see e.g. [8]), we define the extended phase space for dynamical system (3.1) as follows

$$
\Phi_{\varepsilon}:=B_{R_{0}(\varepsilon)} \times \mathcal{H}\left(f_{\varepsilon}\right)
$$

(where the hull $\mathcal{H}\left(f_{\varepsilon}\right)$ is endowed by the topology of $C_{b}\left(\mathbb{R}_{+}, C_{l o c}^{3}(\mathbb{R})\right)$ ) and define the extended semigroup $\mathbb{S}_{t}^{\varepsilon}$ associated with problems $(2.3)$ on $\Phi_{\varepsilon}$ via

$$
\mathbb{S}_{t}^{\varepsilon}\left(\xi^{0}, \phi_{\varepsilon}\right):=\left(U_{\phi_{\varepsilon}}(t, 0) \xi^{0}, T_{t} \phi_{\varepsilon}\right), \quad \xi^{0} \in B_{R_{0}(\varepsilon)}, \phi_{\varepsilon} \in \mathcal{H}\left(f_{\varepsilon}\right) .
$$


It is well known that (3.3) generates indeed a semigroup in $\Phi_{\varepsilon}$, therefore, we may consider its global attractor.

Definition 3.1. The set $\mathbb{A}_{\varepsilon} \subset \Phi_{\varepsilon}$ is a (global) attractor of semigroup (3.3) if

1) The set $\mathbb{A}_{\varepsilon}$ is compact in $\Phi_{\varepsilon}$;

2) This set is strictly invariant, i.e. $\mathbb{S}_{t}^{\varepsilon} \mathbb{A}_{\varepsilon}=\mathbb{A}_{\varepsilon}$;

3) This set attracts all (bounded) subsets of $\Phi_{\varepsilon}$, i.e., for every $B \subset \Phi_{\varepsilon}$ and every neighborhood $\mathcal{O}\left(\mathbb{A}_{\varepsilon}\right)$ of the attractor $\mathbb{A}_{\varepsilon}$ in $\Phi_{\varepsilon}$, there exists $T=T(B, \mathcal{O})$ such that

$$
\mathbb{S}_{t}^{\varepsilon} B \subset \mathcal{O}\left(\mathbb{A}_{\varepsilon}\right), \text { for every } t \geq T \text {. }
$$

If the set $\mathbb{A}_{\varepsilon}$ is the global attractor of the extended semigroup (3.3) then, by definition, the uniform attractor $\mathcal{A}_{\varepsilon}$ of the family (3.1) is a projection of $\mathbb{A}_{\varepsilon}$ to the first component of the Cartesian product $\Phi_{\varepsilon}=B_{R_{0}(\varepsilon)} \times \mathcal{H}\left(f_{\varepsilon}\right)$ :

$$
\mathcal{A}_{\varepsilon}=\Pi_{1} \mathbb{A}_{\varepsilon}
$$

see [8] for the details.

The following theorem establishes the existence of the uniform attractor $\mathcal{A}_{\varepsilon}$ for the family (3.1) associated with nonautonomous hyperbolic equations (2.3).

Theorem 3.2. Let the assumptions of Corollary 2.6 hold. Then, semigroup (3.3) possesses a global attractor $\mathbb{A}_{\varepsilon}$ and, consequently, each dynamical processes (3.1) possess a uniform attractor $\mathcal{A}_{\varepsilon}$. Moreover, these attractors are uniformly (with respect to $\varepsilon$ ) bounded in the space $E^{1}(\Omega)$, i.e.

$$
\left\|\mathcal{A}_{\varepsilon}\right\|_{E^{1}(\Omega)} \leq \bar{R}, \quad \text { for every } \varepsilon \in\left[0, \varepsilon_{0}\right]
$$

and possess the following description:

$$
\mathcal{A}_{\varepsilon}=\cup_{\phi_{\varepsilon} \in \mathcal{H}\left(f_{\varepsilon}\right)} \Pi_{t=0} \mathcal{K}_{\phi_{\varepsilon}}
$$

where $\mathcal{K}_{\phi_{\varepsilon}}$ is a union of all solutions $u(t)$ of equation (2.3) (with the fixed nonlinearity $\left.\phi_{\varepsilon} \in \mathcal{H}\left(f_{\varepsilon}\right)\right)$ which are defined for every $t \in \mathbb{R}$ and satisfy $\left\|\xi_{u}(t)\right\|_{E^{1}(\Omega)} \leq \bar{R}$, for every $t \in \mathbb{R}\left(\mathcal{K}_{\phi_{\varepsilon}}\right.$ is a kernel of equation (2.3) in the terminology of [8]).

Proof. According to the existence theorem for the global and uniform attractors, see [8] or [29], we need to verify the following conditions:

1) Operators (3.3) are continuous in $\Phi_{\varepsilon}$ for every fixed $t$;

2) There exists a compact attracting set $\mathcal{B}_{\varepsilon} \subset B_{\bar{R}} \times \mathcal{H}\left(f_{\varepsilon}\right)$ of this semigroup.

Moreover, the first condition of this theorem is obvious (see Proposition 2.7), so there only remains to construct the compact attracting set $\mathcal{B}_{\varepsilon}$. To this end, we first note that, due to estimate $(2.21)$, the set

$$
\tilde{\mathcal{B}}_{\varepsilon}:=B_{\bar{R}} \times \mathcal{H}\left(f_{\varepsilon}\right),
$$

where $B_{\bar{R}}$ be the $\bar{R}$ ball of the space $E^{1}(\Omega)$, will be an absorbing set for semigroup (3.3) if $\bar{R}$ is large enough (which is, however, not compact in $\Phi_{\varepsilon}$ ). In order to construct the compact analogue of (3.8), we split an arbitrary solution $u$ of (2.3) with the initial data belonging to $B_{\bar{R}}$ as a sum of three functions: $u(t)=G+v(t)+$ $w(t)$, where $G$ solves the linear elliptic problem

$$
-\Delta_{x} G+\lambda_{0} G=\underset{17}{g},\left.\quad \partial_{n} G\right|_{\partial \Omega}=0
$$


the function $v(t)$ solves the linear homogeneous hyperbolic problem

$$
\partial_{t}^{2} v+\gamma \partial_{t} v-\Delta_{x} v+\lambda_{0} v=0,\left.\quad \xi_{v}\right|_{t=\tau}=\left.\xi_{u}\right|_{t=\tau}
$$

and the remainder $w(t)$ is a solution of

$$
\partial_{t}^{2} w+\gamma \partial_{t} w-\Delta_{x} w+\lambda_{0} w=h_{u}(t):=-\phi_{\varepsilon}(u(t), t),\left.\xi_{w}\right|_{t=\tau}=0 .
$$

Then, obviously $G \in H^{2}(\Omega)$ and, consequently, $\xi_{G}:=(G, 0) \in E^{1}(\Omega)$ and, thanks to Proposition A.1, we have

$$
\left\|\xi_{v}(t)\right\|_{E^{1}(\Omega)} \leq C e^{-\alpha(t-\tau)}\left\|\xi_{u}(0)\right\|_{E^{1}(\Omega)},
$$

for some positive $\alpha$. Moreover, due to estimates (1.1), (2.28), Lemma 1.3 and the fact that $\xi_{u}(\tau) \in B_{\bar{R}}$, we have

$$
\left\|h_{u}(t)\right\|_{H^{2}(\Omega)} \leq C
$$

where $C$ is independent of $\varepsilon, \xi_{u}(\tau), t$ and $\phi_{\varepsilon}$. Thus, applying Proposition A.1 to equation (3.11), we derive that

$$
\left\|\xi_{w}(t)\right\|_{E^{1+\delta}(\Omega)} \leq \bar{R}_{1}
$$

where $0<\delta<1 / 2$ and the constant $\bar{R}_{1}$ which is independent of $\varepsilon, t, \tau$ and $\phi_{\varepsilon}$. Estimates (3.12) and (3.14) show that the set

$$
B_{\bar{R}_{1}, \delta}(G):=\xi_{G}+\left\{\xi \in E^{1+\delta}(\Omega),\|\xi\|_{E^{1+\delta}(\Omega)} \leq \bar{R}_{1}\right\}
$$

is a uniform (with respect to $t, \tau$ and $\phi_{\varepsilon}$ ) attracting set for family of the processes (3.1). Thus, taking into account that the hull $\mathcal{H}\left(f_{\varepsilon}\right)$ is compact in $C_{b}\left(\mathbb{R}, C_{\text {loc }}^{3}(\mathbb{R})\right)$ (due to the almost-periodicity of $f_{\varepsilon}$, see Definition 1.2), we finally derive that the set $\mathcal{B}_{\varepsilon}:=B_{\bar{R}_{1}, \delta}(G) \times \mathcal{H}\left(f_{\varepsilon}\right)$ is a compact attracting set for semigroup (3.3) in $\Phi_{\varepsilon}$.

Therefore, all the assumptions of the attractor existence theorem are verified for the semigroup (3.3) and, consequently, this semigroup indeed possesses the global attractor $\mathbb{A}_{\varepsilon} \subset \mathcal{B}_{\varepsilon}$. It remains to note that estimate (3.6) is an immediate corollary of this embedding and our construction of the set $\mathcal{B}_{\varepsilon}$ and description (3.7) is a standard corollary of the abstract attractors existence theorem mentioned above, see [8]. Theorem 3.2 is proven.

Remark 3.3. There exists an intrinsic definition of the uniform attractor $\mathcal{A}_{\varepsilon}$ which does not use the extended semigroup $\mathbb{S}_{t}^{\varepsilon}$, namely, the set $\mathcal{A}_{\varepsilon}$ is a uniform attractor for equation (0.1) if the following conditions are satisfied:

1) $\mathcal{A}_{\varepsilon}$ is compact in $E^{1}(\Omega)$;

2) $\mathcal{A}_{\varepsilon}$ is a uniform (with respect to $\tau \in \mathbb{R}$ ) attracting set of (3.1), i.e., for every (bounded) $B \subset B_{R_{0}(\varepsilon)}$, we have

$$
\lim _{t \rightarrow \infty} \sup _{\tau \in \mathbb{R}} \operatorname{dist}_{E^{1}(\Omega)}\left(U_{f_{\varepsilon}}(\tau+t, \tau) B, \mathcal{A}_{\varepsilon}\right)=0,
$$

where $\operatorname{dist}_{V}(X, Y)$ denotes the non-symmetric Hausdorff distance between sets $X$ and $Y$ in a metric space $V$;

3) The set $\mathcal{A}_{\varepsilon}$ is a minimal compact set which satisfies properties 1) and 2).

The equivalence of this definition and Definition 3.1 is proved in [8].

We now recall, that in the limit case $\varepsilon=0$, we have the autonomous equation (2.1) which generates semigroup (2.30) on the whole space $E^{1}(\Omega)$ and, consequently, has a global attractor $\mathcal{A}_{0}$. The next result shows that, in a sense, this attractor can be interpreted as the average of attractors $\mathcal{A}_{\varepsilon}$. 
Corollary 3.4. Let the assumptions of Theorem 3.2 hold. Then the family $\mathcal{A}_{\varepsilon}$, $\varepsilon \in\left[0, \varepsilon_{0}\right]$ is upper semicontinuous at $\varepsilon=0$, i.e., for every neighborhood $\mathcal{O}\left(\mathcal{A}_{0}\right)$ in $E^{1}(\Omega)$, there exists $\varepsilon^{\prime}=\varepsilon^{\prime}(\mathcal{O})$ such that

$$
\mathcal{A}_{\varepsilon} \subset \mathcal{O}\left(\mathcal{A}_{0}\right) \text { if } \varepsilon \leq \varepsilon^{\prime}
$$

Indeed, thanks to Proposition 2.8, family (3.1) of the dynamical processes associated with equation (2.3) tends uniformly (with respect to $\phi_{\varepsilon} \in \mathcal{H}\left(f_{\varepsilon}\right)$ ) as $\varepsilon \rightarrow 0$ to the limit semigroup (2.30) associated with the limit autonomous equation (2.1). Thus, semicontinuity (3.16) is an immediate corollary of estimate (3.6) and the abstract theorem on the upper semicontinuity of the global (uniform) attractors, see e.g., [3] and [8] (see also the proof of Corollary 3.5 below).

We now discuss the rate of convergence of the non-averaged attractors $\mathcal{A}_{\varepsilon}$ to the averaged one $\mathcal{A}_{0}$ (in the sense of the upper semicontinuity) as $\varepsilon \rightarrow 0$. We recall that this rate of convergence essentially depends on the rate of attraction to the limit attractor $\mathcal{A}_{0}$ and, since this rate of attraction can be arbitrarily slow in general, we cannot obtain the estimates of the rate of convergence of $\mathcal{A}_{\varepsilon}$ to $\mathcal{A}_{0}$ without the additional assumptions on the limit attractor $\mathcal{A}_{0}$. One of the most natural additional assumptions is that the limit global attractor $\mathcal{A}_{0}$ is exponential, see [3], [13], [14]. The latter means that, there exists a positive constant $\alpha>0$ and the monotonic function $Q$ such that, for every bounded subset $B \subset E^{1}(\Omega)$, we have

$$
\operatorname{dist}_{E^{1}(\Omega)}\left(S_{t} B, \mathcal{A}_{0}\right) \leq Q\left(\|B\|_{E^{1}(\Omega)}\right) e^{-\alpha t} .
$$

Here and below, the symbol $\operatorname{dist}_{V}(X, Y)$ denotes the nonsymmetric Hausdorff semidistance between sets $X$ and $Y$ in a metric space $V$.

Corollary 3.5. Let the assumptions of Theorem 3.2 hold and let, in addition, estimate (3.17) be satisfied. Then, the following estimate holds:

$$
\operatorname{dist}_{E^{1}(\Omega)}\left(\mathcal{A}_{\varepsilon}, \mathcal{A}_{0}\right) \leq C \alpha_{C_{\bar{R}}}(\varepsilon)^{\kappa}
$$

where the positive constants $C$ and $\kappa$ are independent of $\varepsilon, \bar{R}$ is the same as in Theorem 3.2 and the function $\alpha_{C_{R}}(\varepsilon)$ is the same as in (2.34).

Proof. Indeed, let $\varepsilon>0$ be small enough and $\xi \in \mathcal{A}_{\varepsilon}$ be an arbitrary point. Then, thanks to description (3.7) there exist $\phi_{\varepsilon} \in \mathcal{H}\left(f_{\varepsilon}\right)$ and a solution $u_{\varepsilon}(t), t \in \mathbb{R}$, of equation (2.3) such that $\xi_{u_{\varepsilon}} \in \mathcal{K}_{\phi_{\varepsilon}}$ and $\xi_{u_{\varepsilon}}(0)=\xi$. Fix now an arbitrary $T>0$ and consider a solution $\bar{u}(t), t \geq-T$ such that $\xi_{u_{\varepsilon}}(-T)=\xi_{\bar{u}}(-T)$. Then, on the one hand, thanks to Proposition 2.8 and estimate (3.6), we have

$$
\left\|\xi-\xi_{\bar{u}}(0)\right\|_{E^{1}(\Omega)} \leq C_{\bar{R}} e^{K T} \alpha_{C_{\bar{R}}}(\varepsilon)^{\beta}
$$

and, on the other hand, thanks to (3.17), we have

$$
\operatorname{dist}_{E^{1}(\Omega)}\left(\xi_{\bar{u}}, \mathcal{A}_{0}\right) \leq C_{\bar{R}} e^{-\alpha T} .
$$

Combining (3.19) and (3.20) and taking into account that $\xi \in \mathcal{A}_{\varepsilon}$ is arbitrary, we derive that

$$
\operatorname{dist}_{E^{1}(\Omega)}\left(\mathcal{A}_{\varepsilon}, \mathcal{A}_{0}\right) \leq C_{\bar{R}}\left(e^{-\alpha T}+e^{K T} \alpha_{C_{\bar{R}}}(\varepsilon)^{\beta}\right) .
$$


Optimizing the right-hand side of (3.21) with respect to $T$ (i.e., fixing $T=T(\varepsilon)$ as a solution of $\left.e^{-\alpha T}=e^{K T} \alpha_{C_{\bar{R}}}(\varepsilon)^{\beta}\right)$, we derive estimate (3.18) and finish the proof of Corollary 3.5.

We now recall that assumption (3.17) is satisfied for generic external forces $g \in$ $L^{2}(\Omega)$ (for which all of the equilibria of equation (2.1) are hyperbolic, see [3]). In this case, the limit attractor $\mathcal{A}_{0}$ has a specific structure (the so-called regular attractor in the terminology of [3], see also Section 5 below) which allows to prove, for instance, that the family of attractors $\mathcal{A}_{\varepsilon}$ is also lower semicontinuous as $\varepsilon=0$ and to obtain the analogue of (3.18) for the symmetric Hausdorff distance. However, in order to study the non-autonomous perturbations of regular attractors, it is more convenient to use the alternative concept of the pullback attractor.

Definition 3.6. Let $\{U(t, \tau), \tau \in \mathbb{R}, t \geq \tau\}$ be a dynamical process in a metric space $\Psi$ which satisfies the cocycle property:

$$
U(t, \tau) \circ U(\tau, s)=U(t, s), \quad t \geq s \geq \tau
$$

Then, the set-valued function $t \rightarrow \mathcal{A}(t)$ is a pullback attractor of this process if the following conditions hold:

1) The sets $\mathcal{A}(t) \subset \Psi$ are compact for every $t \in \mathbb{R}$;

2) The sets $\mathcal{A}(t)$ are strictly invariant, i.e. $U(t, \tau) \mathcal{A}(\tau)=\mathcal{A}(t)$.

3) The pullback attraction property is satisfied, i.e., for every bounded subset $B \subset \Psi$ and every $t \in \mathbb{R}$, we have

$$
\lim _{T \rightarrow+\infty} \operatorname{dist}_{E^{1}(\Omega)}(U(t, t-T) B, \mathcal{A}(t))=0 .
$$

Corollary 3.7. Let the assumptions of Theorem 3.2 hold. Then, for every $\varepsilon \leq \varepsilon_{0}$ and every $\phi_{\varepsilon} \in \mathcal{H}\left(f_{\varepsilon}\right)$, dynamical process (3.1) possesses the pullback attractor $\mathcal{A}_{\phi_{\varepsilon}}(t)$ which has the following structure:

$$
\mathcal{A}_{\phi_{\varepsilon}}(\tau)=\Pi_{t=\tau} \mathcal{K}_{\phi_{\varepsilon}}
$$

where the sets $\mathcal{K}_{\phi_{\varepsilon}}$ are defined in Theorem 3.2.

Indeed, according to the general theory (see [8]), the existence of the uniform attractor $\mathcal{A}_{\varepsilon}$ implies the existence of the pullback attractors $\mathcal{A}_{\phi_{\varepsilon}}(t)$ for every dynamical process $U_{\phi_{\varepsilon}}(t, \tau), \phi_{\varepsilon} \in \mathcal{H}\left(f_{\varepsilon}\right)$, and relation (3.24).

Remark 3.8. In general, the convergence in (3.23) is not uniform with respect to $t \in \mathbb{R}$ and (consequently) the sets $U_{\phi_{\varepsilon}}(t+T, t) B$ are not necessarily converge to $\mathcal{A}_{\phi_{\varepsilon}}(t+T)$ as $T \rightarrow+\infty$. Nevertheless, in contrast to what generally happens, we prove in Section 5 below that we have this convergence (which will be even exponential) in the case where the limit attractor $\mathcal{A}_{0}$ is regular and $\varepsilon>0$ is small enough.

We also mention the following obvious, but useful, relation between the uniform and pullback attractors:

$$
\mathcal{A}_{\varepsilon}=\cup_{\phi_{\varepsilon} \in \mathcal{H}\left(f_{\varepsilon}\right)} \mathcal{A}_{\phi_{\varepsilon}}(0)
$$

which is an immediate corollary of (3.7) and (3.24). 


\section{$\S 4$ Averaging near the hyperbolic equilibrium.}

In this section, we investigate the behavior of solutions of the non-averaged system (2.3) in a small neighborhood of the hyperbolic equilibrium of the averaged system (2.1) if $\varepsilon>0$ is small enough. In particular, we construct here the nonautonomous unstable manifold associated with this equilibrium which is necessary for studying (in the next section) the non-autonomous perturbations of the averaged regular attractor $\mathcal{A}_{0}$. Since all the results of this section are more or less standard corollaries of Propositions 2.7 and 2.8 and the implicit function theorem, we only give below the necessary definitions and statements and indicate the main ideas of their proofs leaving the details to the reader (see also [14] and [18]).

We assume from now on that $\xi_{z_{0}}:=\left(z_{0}, 0\right), z_{0}=z_{0}(x)$ is a hyperbolic equilibrium of equation (2.1), i.e.

$$
-\Delta_{x} z_{0}+\lambda_{0} z_{0}+\bar{f}\left(z_{0}\right)=g,\left.\quad z_{0}\right|_{\partial \Omega}=0
$$

and the spectrum of the linearization of (4.1) near $\xi_{z_{0}}$ does not contain zero:

$$
0 \notin \sigma\left(-\Delta_{x}+\lambda_{0}+\bar{f}^{\prime}\left(z_{0}\right), L^{2}(\Omega)\right)
$$

Then, according to Proposition 2.7, the Frechet derivative $D_{\xi^{0}} S_{t}\left(\xi_{z_{0}}\right)$ of the semigroup $S_{t}$ generated by the averaged equation (2.1) at $\xi_{z_{0}}$ satisfies the following linear equation

$$
\partial_{t}^{2} v_{\theta}+\gamma \partial_{t} v_{\theta}-\Delta_{x} v_{\theta}+\lambda_{0} v_{\theta}+\bar{f}^{\prime}\left(z_{0}\right) v_{\theta}=0,\left.\quad \xi_{v_{\theta}}\right|_{t=0}=\theta
$$

where $\theta \in E^{1}(\Omega)$ and $v_{\theta}:=\left[D_{\xi^{0}} S_{t}\left(\xi_{z_{0}}\right)\right] \theta$. Moreover, hyperbolicity assumption (4.2) implies the existence of an exponential dichotomy for equation (4.3), i.e. there exist two subspaces $E_{ \pm}$of $E^{1}(\Omega)$ such that

$$
E^{1}(\Omega)=E_{+} \oplus E_{-}, \quad D_{\xi^{0}} S_{t}\left(\xi_{z_{0}}\right) E_{ \pm}=E_{ \pm}, \quad \operatorname{dim} E_{+}=\operatorname{ind}^{+}\left(z_{0}\right)<\infty
$$

and there exist positive constants $C$ and $\alpha$ such that, for every $t \geq 0$

$$
\left\{\begin{array}{l}
\left\|D_{\xi^{0}} S_{t}\left(z_{0}\right) \theta\right\|_{E^{1}(\Omega)} \leq C e^{-\alpha t}\|\theta\|_{E^{1}(\Omega)}, \quad \forall \theta \in E_{-}, \\
\left\|D_{\xi^{0}} S_{t}\left(z_{0}\right) \theta\right\|_{E^{1}(\Omega)} \geq C^{-1} e^{+\alpha t}\|\theta\|_{E^{1}(\Omega)}, \quad \forall \theta \in E_{+},
\end{array}\right.
$$

see e.g., [3]. We denote by $\Pi_{ \pm}: E^{1}(\Omega) \rightarrow E_{ \pm}$the spectral projectors associated with decomposition (4.5).

The main task of this section is to obtain the nonlinear and non-autonomous analogue of decomposition (4.4) for the case of equation (2.3) in a small neighborhood of $z_{0}$. To this end, we first construct the analogue of the equilibrium $z_{0}$ for equation (2.3).

Theorem 4.1. Let the assumptions of Theorem 3.2 hold and let $\xi_{z_{0}}$ be a hyperbolic equilibrium of equation (2.1). Then there exist $\varepsilon_{0}>0$ and a small neighborhood $V_{z_{0}}$ of the equilibrium $\xi_{z_{0}}$ in $E^{1}(\Omega)$ such that, for every $\varepsilon<\varepsilon_{0}$ and $\phi_{\varepsilon} \in \mathcal{H}\left(f_{\varepsilon}\right)$, equation (2.3) possesses a unique solution $u_{\phi_{\varepsilon}, z_{0}}(t), t \in \mathbb{R}$ such that

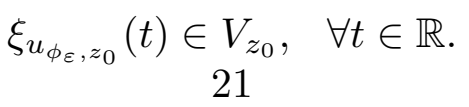


Moreover, this solution is almost-periodic with respect to $t$ (as a $E^{1}(\Omega)$-valued function) with the same frequency basis as the nonlinearity $f_{\varepsilon}$ and tends to $\xi_{z_{0}}$ as $\varepsilon \rightarrow 0$ :

$$
\left\|\xi_{u_{\phi_{\varepsilon}, z_{0}}}(t)-\xi_{z_{0}}\right\|_{E^{1}(\Omega)} \leq C \alpha_{C_{\bar{R}}}(\varepsilon)^{\beta}, \forall t \in \mathbb{R}
$$

where $\bar{R}$ is the same as in Theorem 3.2 the function $\alpha_{C_{\bar{R}}}(\varepsilon)^{\beta}$ is the same as in Proposition 2.8 and the constant $C$ is independent of $\varepsilon, t$, and $\phi_{\varepsilon}$.

Sketch of the proof. Following [14], instead of solving (2.3), we solve the equivalent difference equation

$$
\xi(n)=U_{\phi_{\varepsilon}}(n, n-1) \xi(n-1), \quad n \in \mathbb{Z}
$$

in the space $\mathbb{L}\left(E^{1}\right):=L^{\infty}\left(\mathbb{Z}, E^{1}(\Omega)\right)$ of $E^{1}(\Omega)$-valued sequences. To this end, we introduce an operator

$$
\Phi: \mathbb{L}\left(E^{1}\right) \times \mathbb{R}_{+} \rightarrow \mathbb{L}\left(E^{1}\right), \quad \Phi(\xi, \varepsilon)(n):=\xi(n)-U_{\phi_{\varepsilon}}(n, n-1) \xi(n-1) .
$$

Then, due to Propositions 2.7 and 2.8, the operators $U_{\phi_{\varepsilon}}(n, n-1)$ tend together with the Frechet derivative to the solving operator $S_{1}$ of the averaged equation (2.1) (uniformly with respect to $n$ and $\phi_{\varepsilon}$ ). Thus, $\Phi\left(\xi_{z_{0}}, 0\right)=0$. Consequently, in order to apply the implicit function theorem to (4.9), it remains to verify that the derivative

$$
\left(D_{\xi} \Phi\left(\xi_{z_{0}}, 0\right) \psi\right)(n)=\psi(n)-D_{\xi^{0}} S_{1}\left(\xi_{z_{0}}\right) \psi(n-1)
$$

is invertible in $\mathbb{L}\left(E^{1}\right)$, but this fact is a standard corollary of exponential dichotomy (4.5). Thus, thanks to the implicit function theorem, there exists a neighborhood $V_{z_{0}}$ of $\xi_{z_{0}}$ and $\varepsilon_{0}>0$ such that, for every $\varepsilon<\varepsilon_{0}$ and $\phi_{\varepsilon} \in \mathcal{H}\left(f_{\varepsilon}\right)$, there exists a unique sequence $\xi(n)=\xi_{\phi_{\varepsilon}, z_{0}}(n)$ which belongs to $V_{z_{0}}$, solves (4.8), satisfies (4.7) for $t \in \mathbb{Z}$ and depends continuously on $\phi_{\varepsilon} \in \mathcal{H}\left(f_{\varepsilon}\right)$ (the detailed derivation of this fact is given in [14]). Moreover, since $\xi_{\phi_{\varepsilon}, z_{0}}$ is unique, then translation identity (2.29) implies that

$$
U_{\phi_{\varepsilon}}(n+t, n) \xi_{\phi_{\varepsilon}, z_{0}}(n)=\xi_{T_{t} \phi_{\varepsilon}, z_{0}}(n), \forall t \in \mathbb{R}_{+} .
$$

Then, the required continuous solution $u_{\phi_{\varepsilon}, z_{0}}(t)$ can be defined via

$$
u_{\phi_{\varepsilon}, z_{0}}(t):=u_{T_{t} \phi_{\varepsilon}, z_{0}}(0), \quad t \in \mathbb{R} .
$$

Indeed, the fact that (4.12) solves (2.3) follows from (4.11) and the almost-periodicity of (4.12) is an immediate corollary of the fact that the flow $T_{t}$ is almost-periodic on the hull $\mathcal{H}\left(f_{\varepsilon}\right)$, see [24]. Theorem 4.1 is proven.

We are now ready to define the nonlinear analogue of the space $E_{+}$for equation (2.3).

Definition 4.2. Let the assumptions of Theorem 4.1 hold and let $\tilde{V}_{z_{0}}$ be a sufficiently small neighborhood of $\xi_{z_{0}}$ in $E^{1}(\Omega)$. Then, for every $\varepsilon \leq \varepsilon_{0}, \phi_{\varepsilon} \in \mathcal{H}\left(f_{\varepsilon}\right)$ and $\tau \in \mathbb{R}$, we define the unstable set $\mathcal{M}_{\phi_{\varepsilon}, z_{0}}^{+, l o c}(t)$ as follows:

$$
\begin{aligned}
\mathcal{M}_{\phi_{\varepsilon}, z_{0}}^{+, l o c}(\tau):=\left\{\xi^{\tau} \in E^{1}(\Omega), \quad \exists \xi_{u_{\varepsilon}} \in \mathcal{K}_{\phi_{\varepsilon}}\right. \\
\text { such that } \left.\xi_{u_{\varepsilon}}(\tau)=\xi^{\tau} \text { and } \xi_{u_{\varepsilon}}(t) \in \tilde{V}_{z_{0}} \quad \forall t \leq \tau\right\} .
\end{aligned}
$$

The following theorem shows that sets (4.13) are finite-dimensional manifolds if $\varepsilon$ is small enough. 
Theorem 4.3. Let the assumptions of Theorem 4.1 hold. Then, there exist a neighborhood $\tilde{V}_{z_{0}}$ of the equilibrium $\xi_{z_{0}}$ and $\varepsilon_{0}^{\prime}>0$ such that the sets (4.13) are finite-dimensional submanifolds of $E^{1}(\Omega)$, for every $\varepsilon \leq \varepsilon_{0}^{\prime}, \phi_{\varepsilon} \in \mathcal{H}\left(f_{\varepsilon}\right)$ and $\tau \in \mathbb{R}$. To be more precise, there exist a neighborhood $W^{+} \subset E_{+}$of zero in $E_{+}$(which is independent of $\varepsilon, \tau$ and $\left.\phi_{\varepsilon}\right)$, a family of neighborhoods $W_{\phi_{\varepsilon}}^{+}(\tau)$ of zero in $E_{+}$such that $W^{+} \subset W_{\phi_{\varepsilon}}^{+}(\tau)$ and a family of $C^{1}$-maps

$$
\mathbb{M}_{\phi_{\varepsilon}, \tau}^{+}: W_{\phi_{\varepsilon}, \tau}^{+} \rightarrow E_{-}
$$

such that

$$
\mathcal{M}_{\phi_{\varepsilon}, z_{0}}^{+, l o c}(\tau)=u_{\phi_{\varepsilon}, z_{0}}(\tau)+\left\{\xi^{+}+\mathbb{M}_{\phi_{\varepsilon}, \tau}^{+}\left(\xi^{+}\right), \quad \xi^{+} \in W_{\phi_{\varepsilon}}^{+}(\tau)\right\}
$$

and

$$
\left\|\mathbb{M}_{\phi_{\varepsilon}, \tau}^{+}\right\|_{C^{1}} \leq C, \quad \mathbb{M}_{\phi_{\varepsilon}, \tau}^{+}(0)=0
$$

where the constant $C$ is independent of $\varepsilon, \tau$ and $\phi_{\varepsilon}$. Moreover, for every $\xi^{\tau} \in$ $\mathcal{M}_{\phi_{\varepsilon}, z_{0}}^{+, l o c}(\tau)$, the corresponding solution $\xi_{u_{\varepsilon}} \in \mathcal{K}_{\phi_{\varepsilon}}$ (which exists due to definition (4.13)) tends exponentially as $t \rightarrow-\infty$ to the almost-periodic solution $\xi_{u_{\phi_{\varepsilon}, z_{0}}}$ :

$$
\left\|\xi_{u_{\varepsilon}}(t)-\xi_{u_{\phi_{\varepsilon}, z_{0}}}(t)\right\|_{E^{1}(\Omega)} \leq C e^{\alpha(t-\tau)}\left\|\xi-\xi_{u_{\phi_{\varepsilon}, z_{0}}}\right\|_{E^{1}(\Omega)}, \quad t \leq \tau
$$

where the positive constants $C$ and $\alpha$ are independent of $\varepsilon, \xi, \phi_{\varepsilon}$ and $\tau$.

Sketch of the proof. By definition (4.13), in order to construct the unstable manifold $\mathcal{M}_{\phi_{\varepsilon}, z_{0}}^{+, l o c}(\tau)$, it is sufficient to find all the backward solutions of equation (2.3) defined for $t \leq \tau$ and belonging to the small neighborhood $\tilde{V}_{z_{0}}$ of $\xi_{z_{0}}$, and to prove that the set of all these solutions generates a manifold. Moreover, without loss of generality, we may assume that $\tau=0$. The general case reduces to this particular one using the obvious translation formula

$$
\mathcal{M}_{\phi_{\varepsilon}, z_{0}}^{+, l o c}(\tau)=\mathcal{M}_{T_{\tau} \phi_{\varepsilon}, z_{0}}^{+, l o c}(0) .
$$

As in the proof of Theorem 4.1, instead of finding the backward solutions of problem (2.3), it is more convenient to solve the equivalent difference equation (4.8) on the space of one-sided sequences $\mathbb{L}^{-}\left(E^{1}\right):=L^{\infty}\left(\mathbb{Z}_{-}, E^{1}(\Omega)\right)$, but, in contrast to the proof of Theorem 4.1, we now need to endow equation (4.1) with the appropriate initial condition at $n=0$. To be more precise, we make the change of variables $\tilde{\xi}(n):=\xi(n)-\xi_{u_{\phi_{\varepsilon}, z_{0}}}(n)$ and we consider, for every $\xi^{+} \in E_{+}$, the following problem in the space $\mathbb{L}^{-}\left(E^{1}\right)$ :

$$
\begin{aligned}
\tilde{\xi}(n)=U_{\phi_{\varepsilon}}(n, n-1)\left(\xi_{u_{\phi_{\varepsilon}, z_{0}}}(n-1)+\tilde{\xi}(n-1)\right)- & \\
- & U_{\phi_{\varepsilon}}(n, n-1)\left(\xi_{u_{\phi_{\varepsilon}, z_{0}}}(n-1)\right), \quad \Pi_{+} \tilde{\xi}(0)=\xi^{+} .
\end{aligned}
$$

As in the proof of Theorem 4.1, the uniform convergence of operators $U_{\phi_{\varepsilon}}(n, n-1)$ to the limit semigroup $S_{1}$ established in Propositions 2.7 and 2.8 and the exponential dichotomy (4.5) allow us to prove, using the implicit function theorem, that for sufficiently small $\varepsilon \leq \varepsilon_{0}^{\prime}$, equation (4.19) possesses a unique solution $\xi_{v_{\phi_{\varepsilon}, \xi^{+}}}(n)$, 
$n \leq 0$, which belongs to $\tilde{V}_{z_{0}}$ and this solution depends smoothly $\left(C^{1}\right)$ on the initial data $\xi^{+}$belonging to some small neighborhood $W_{\phi_{\varepsilon}}^{+}$of zero in $E^{+}$(the detailed proof of this fact is given in [14]). Thus, the desired maps $\mathbb{M}_{\phi_{\varepsilon}, 0}^{+}\left(\xi^{+}\right)$can be now defined via

$$
\mathbb{M}_{\phi_{\varepsilon}, 0}^{+}\left(\xi^{+}\right):=\Pi_{-} \xi_{v_{\phi_{\varepsilon}, \xi^{+}}}(0), \quad \xi^{+} \in W_{\phi_{\varepsilon}}^{+} .
$$

Indeed, the representation (4.15) is an immediate corollary of the construction of the solution $\xi_{v_{\phi_{\varepsilon}, \xi^{+}}}$and estimate (4.16) follows from (4.19) and the implicit function theorem. Thus, there only remains to verify (4.17) or (which is the same) that the constructed solution $v_{\phi_{\varepsilon}, \xi^{+}}(n)$ of equation (4.19) decays exponentially as $n \rightarrow-\infty$. To this end, following [14], it is sufficient to consider equation (4.19) in the weighted space $\mathbb{L}_{\beta}^{-}\left(E^{1}\right)$ of sequences decaying exponentially as $n \rightarrow-\infty$ (the norm of this space is given by $\left.\|\xi\|_{\mathbb{L}_{\beta}^{-}\left(E^{1}\right)}:=\sup _{n \leq 0} e^{-\beta n}\|\xi(n)\|_{E^{1}(\Omega)}, \beta>0\right)$. As shown in [14], the exponential dichotomy (4.5) allows to apply the implicit function theorem to equation (4.19) not only in the space $\mathbb{L}^{-}\left(E^{1}\right)$, but also in $\mathbb{L}_{\beta}^{-}$for $\beta>0$ small enough, and obtain a solution $\xi_{\tilde{v}_{\phi_{\varepsilon}, \xi^{+}}} \in \mathbb{L}_{\beta}^{-}\left(E^{1}\right)$. Finally, the uniqueness part of the implicit function theorem implies that $\xi_{\tilde{v}_{\phi_{\varepsilon}, \xi^{+}}}=\xi_{v_{\phi_{\varepsilon}, \xi^{+}}}$, therefore, (4.17) is verified and Theorem 4.3 is proven.

Remark 4.4. According to Theorem 4.3, $\operatorname{dim} \mathcal{M}_{\phi_{\varepsilon}, z_{0}}^{+, l o c}(\tau)=\operatorname{dim} E_{+}=\operatorname{ind}^{+}\left(z_{0}\right)$. Moreover, it follows from the proof of Theorem 4.3 that these manifolds are $C^{1}$ diffeomorphic to $\mathbb{R}^{\text {ind }^{+}\left(z_{0}\right)}$. We also note that, analogously to Theorem 4.3 , we may also construct the local stable manifolds $\mathcal{M}_{\phi_{\varepsilon}, z_{0}}^{-, l o c}(\tau)$ which are diffeomorphic to $E_{-}$ and consist of all solutions of (2.3) stabilizing to $u_{\phi_{\varepsilon}, z_{0}}(t)$ as $t \rightarrow+\infty$, but these manifold are not necessary for the construction of regular attractors and, therefore, we do not consider them here.

We are now ready to define the global unstable sets $\mathcal{M}_{\phi_{\varepsilon}, z_{0}}^{+}(\tau)$ via

$$
\begin{aligned}
\mathcal{M}_{\phi_{\varepsilon}, z_{0}}^{+}(\tau):=\left\{\xi^{\tau} \in E^{1}(\Omega), \exists \xi_{u_{\varepsilon}} \in \mathcal{K}_{\phi_{\varepsilon}}\right. \text { such that } \\
\left.\qquad \xi_{u_{\varepsilon}}(\tau)=\xi^{\tau} \text { and } \lim _{t \rightarrow-\infty}\left\|\xi_{u_{\varepsilon}}(t)-\xi_{u_{\phi_{\varepsilon}, z_{0}}}(t)\right\|_{E^{1}(\Omega)}=0\right\}
\end{aligned}
$$

which consist of values at $t=\tau$ of all solutions $\xi_{u} \in \mathcal{K}_{\phi_{\varepsilon}}$ which stabilize to the "equilibrium" $\xi_{u_{\phi_{\varepsilon}, z_{0}}}(t)$ as $t \rightarrow-\infty$. Then, obviously, the sets $\mathcal{M}_{\phi_{\varepsilon}, z_{0}}^{+}(\tau), \tau \in \mathbb{R}$, are strictly invariant with respect to $U_{\phi_{\varepsilon}}(t, \tau)$, i.e.

$$
U_{\phi_{\varepsilon}}(t, \tau) \mathcal{M}_{\phi_{\varepsilon}, z_{0}}^{+}(\tau)=\mathcal{M}_{\phi_{\varepsilon}, z_{0}}^{+}(t), \quad t \geq \tau
$$

Moreover, due to Definition 2.1 and Theorem 4.3, the global unstable sets can be expressed in terms of the local ones via

$$
\mathcal{M}_{\phi_{\varepsilon}, z_{0}}^{+}(\tau)=\cup_{n=1}^{\infty} U_{\phi_{\varepsilon}}(\tau, \tau-n) \mathcal{M}_{\phi_{\varepsilon}, z_{0}}^{+, l o c}(\tau-n) .
$$

if $\varepsilon$ is small enough. It is also worth to mention that, in the limit case $\varepsilon=0$, we have the autonomous equation (2.1) and, consequently, the limit unstable sets $\mathcal{M}_{\bar{f}, z_{0}}^{+}(\tau)$ which correspond to equation $(2.1)$ are independent of $\tau$, i.e.

$$
\mathcal{M}_{\bar{f}, z_{0}}^{+}(\tau) \equiv \mathcal{M}_{\bar{f}, z_{0}}^{+}, \quad \forall \tau \in \mathbb{R} \text { and } S_{t} \mathcal{M}_{\bar{f}, z_{0}}^{+}=\mathcal{M}_{\bar{f}, z_{0}}^{+}
$$


Remark 4.5. We recall that (4.23) and the fact that $\mathcal{M}_{\phi_{\varepsilon}, z_{0}}^{+, \text {loc }}(\tau) \sim \mathbb{R}^{\text {ind }^{+}\left(z_{0}\right)}$ allow to endow the set $\mathcal{M}_{\phi_{\varepsilon}, z_{0}}^{+}(\tau)$ with the structure of a $C^{1}$-manifold diffeomorphic to $\mathbb{R}^{\text {ind }^{+}\left(z_{0}\right)}$. But in contrast to the local ones, in general, these sets may be not $s u b$ manifolds of $E^{1}(\Omega)$ since the recurrent motions (e.g., homolclinic orbits to $u_{\phi_{\varepsilon}}, z_{0}(t)$ ) may exist near $u_{\phi_{\varepsilon}, z_{0}}(t)$. Nevertheless, in our case, the limit equation (2.1) possesses a global Lyapunov function, which does not allow the motions mentioned above to exist if $\varepsilon \geq 0$ is small enough (see Lemma 5.1 below). Thus, as proved e.g. in [14] (see also [3] and [18]), the sets (4.21) are indeed $C^{1}$-submanifolds of $E^{1}(\Omega)$ diffeomorphic to $\mathbb{R}^{\text {ind }^{+}\left(z_{0}\right)}$ if $\varepsilon$ is small enough.

To conclude this section, we formulate the standard fact that every trajectory of equation (2.3) is exponentially attracted to $\mathcal{M}_{\phi_{\varepsilon}}^{+}(t)$ while staying in the neighborhood of $\xi_{z_{0}}$. This is the main technical tool in the proof of the exponential rate of the attraction to the regular attractor, see [3] and [14].

Theorem 4.6. Let the assumptions of Theorem 4.1 hold. Then, there exist $\varepsilon_{0}^{\prime \prime}>0$ and a neighborhood $V_{z_{0}}$ of the equilibrium $\xi_{z_{0}}$ in $E^{1}(\Omega)$ such that if $\varepsilon \leq \varepsilon_{0}^{\prime \prime}, \tau \in \mathbb{R}$ and $\phi_{\varepsilon} \in \mathcal{H}\left(f_{\varepsilon}\right)$ be arbitrary and $\xi_{u}(t), t \geq \tau$ be an arbitrary solution of $(2.3)$ which satisfies

$$
\xi_{u}(t) \in V_{z_{0}}, \forall t \in[\tau, \tau+N]
$$

for some $N \in \overline{\mathbb{N}}\left(N=+\infty\right.$ is allowed), then there exists a solution $\xi_{u^{+}}(t)$ of equation (2.3) such that $\xi_{u^{+}}(t) \in \mathcal{M}_{\phi_{\varepsilon}, z_{0}}^{+, l o c}(t), t \leq \tau+N$, and

$$
\left\|\xi_{u}(t)-\xi_{u^{+}}(t)\right\|_{E^{1}(\Omega)} \leq C e^{-\beta(t-\tau)}\left\|\xi_{u}(\tau)-\xi_{u^{+}}(\tau)\right\|_{E^{1}(\Omega)}, \quad t \in[\tau, \tau+N]
$$

where positive constants $C$ and $\beta$ are independent of $\varepsilon, N, \tau \xi_{u}$ and $\phi_{\varepsilon}$.

The detailed proof of this theorem (which is based on Propositions 2.7 and 2.8 and the dichotomy (4.5)) is given in [14] (in fact, in an abstract setting). This is the reason why we only mention here that the desired solution $\xi_{u^{+}}(t)$ of $(2.3)$ or (which is the same) its discrete analogue $\xi(n)=\xi_{u^{+}}(\tau+n), n \in\{0, \cdots, N\}$ can be obtained applying the implicit function theorem to the following problem:

$$
\left\{\begin{array}{l}
\xi(n)=U_{T_{\tau} \phi_{\varepsilon}}(n, n-1) \xi(n-1), \quad n=1, \cdots, N \\
\Pi_{-}\left(\xi(0)-\xi_{u_{\phi_{\varepsilon}, z_{0}}}(\tau)\right)=\mathbb{M}_{\phi_{\varepsilon}, \tau}^{+}\left(\Pi_{+}\left(\xi(0)-\xi_{u_{\phi_{\varepsilon}, z_{0}}}(\tau)\right)\right), \\
\Pi_{-} \xi(N)=\Pi_{-} \xi_{u}(\tau+N)
\end{array}\right.
$$

and the remaining details are left to the reader.

Remark 4.7. We note that, in the case $N=+\infty$ in Theorem 4.6, we necessarily have $\xi_{u^{+}}(t) \equiv \xi_{u_{\phi_{\varepsilon}, z_{0}}}(t)$ (since, due to (4.17), this is the only solution belonging to the unstable manifold $\mathcal{M}_{\phi_{\varepsilon}, z_{0}}^{+}(t)$ which remains in a small neighborhood of $\xi_{z_{0}}$ for all $t \geq \tau)$. Thus, thanks to Theorem 4.6, every solution $\xi_{u}(t)$ of equation (2.3) which belongs to $V_{z_{0}}$ for every $t \geq \tau$, stabilizes exponentially to $\xi_{u_{\phi_{\varepsilon}}, z_{0}}(t)$ as $t \rightarrow \infty$.

\section{$\S 5$ The Regular Pullback attractor AND its AVERAging.}

This section is devoted to the detailed study of the pullback attractors $\mathcal{A}_{\phi_{\varepsilon}}(t)$ in the case where $\varepsilon>0$ is small enough and the limit attractor $\mathcal{A}_{0}$ is regular using 
the theory of the non-autonomous perturbations of regular attractors developed in [14] (see also [18]).

We start with the limit case $\varepsilon=0$. In this case, as known, equation (2.1) possesses a global Lyapunov function of the form

$$
L\left(\xi_{u}\right):=\int_{\Omega}\left[\left|\partial_{t} u(x)\right|^{2}+\left|\nabla_{x} u(x)\right|^{2}+\lambda_{0}|u(x)|^{2}+2 F(u(x))-2 g(x) u(x)\right] d x
$$

where $F(u):=\int_{0}^{u} \bar{f}(v) d v$, see, e.g. [3].

The main additional assumption of this section is that all of the equilibria of equation (2.1) are hyperbolic, i.e., that all the solutions of equation (4.1) satisfy condition (4.2). In this case, obviously, the set $\mathcal{R}_{0}$ of all the equilibria of (2.1) is finite:

$$
\mathcal{R}_{0}=\left\{\xi_{z_{i}}\right\}_{i=1}^{N} \text { and } z_{i} \text { satisfies (4.1) and (4.2). }
$$

Under the above assumptions, the limit attractor $\mathcal{A}_{0}$ possesses the following description.

Theorem 5.1. Let the assumptions of Theorem 2.1 hold and, in addition, (5.2) be satisfied. Then, the global attractor $\mathcal{A}_{0}$ is a finite collection of the finite-dimensional unstable manifolds $\mathcal{M}_{\bar{f}, z_{0}}^{+}$associated with the equilibria (5.2):

$$
\mathcal{A}_{0}=\cup_{z_{0} \in \mathcal{R}_{0}} \mathcal{M}_{\bar{f}, z_{0}}^{+}, \quad \mathcal{M}_{\bar{f}, z_{0}}^{+} \sim \mathbb{R}^{\operatorname{ind}^{+}\left(z_{0}\right)} .
$$

Furthermore, every solution $\xi_{u} \in \mathcal{K}_{\bar{f}}$ is a heteroclinic orbit between two different equilibria $\xi_{z_{0}^{+}}$and $\xi_{z_{0}^{-}}$belonging to $\mathcal{R}_{0}$ and every solution $\xi_{u}(t)$ of equation (2.1) defined on a semi-interval $[\tau,+\infty)$ tends, as $t \rightarrow \infty$, to one of the equilibria $\xi_{z_{0}} \in$ $\mathcal{R}_{0}$. Moreover, the attractor $\mathcal{A}_{0}$ attracts exponentially all bounded subsets of $E^{1}(\Omega)$, i.e., estimate (3.17) is satisfied.

The proof of this theorem can be found in [3], see also the explanations in the proof of Theorem 5.3 below.

Remark 5.2. We recall that the hyperbolicity assumption (5.2) is generic in the sense that it is satisfied for all external forces $g(x)$ belonging to an open and dense subset of $L^{2}(\Omega)$, see [3].

The main result of this section is the following theorem which gives the analogous description of the pullback attractors $\mathcal{A}_{\phi_{\varepsilon}}(\tau)$ of equations (2.3) for sufficiently small, but positive $\varepsilon$ and establish the upper and lower semicontinuity of them as $\varepsilon \rightarrow 0$.

Theorem 5.3. Let the assumptions of Theorems 3.2 and 5.1 hold. Then, there exists $\varepsilon_{0}>0$ such that, for every $\varepsilon \leq \varepsilon_{0}$, the following assertions are satisfied:

1) For every $\phi_{\varepsilon} \in \mathcal{H}\left(f_{\varepsilon}\right)$, equation (2.3) possesses exactly $N=\# \mathcal{R}_{0}$ different almost-periodic solutions $\xi_{u_{\phi_{\varepsilon}, z_{i}}}(t), i=1, \cdots, N$, in the ball $B_{R_{0}(\varepsilon)}$ which are constructed in Theorem 4.1.

2) Every complete bounded solution $\xi_{u} \in \mathcal{K}_{\phi_{\varepsilon}}$ of equation (2.3) is a heteroclinic orbit between two different almost-periodic solutions mentioned above, i.e.

$$
\lim _{t \rightarrow \pm \infty}\left\|\xi_{u}(t)-\xi_{u_{\phi_{\varepsilon}, z_{ \pm}}}(t)\right\|_{E^{1}(\Omega)}=0, \quad \xi_{z_{ \pm}} \in \mathcal{R}_{0}, \quad z_{+} \neq z_{-}
$$


and every solution $\xi_{u}(t)$ of (2.3) defined on a semi-interval $t \in[\tau,+\infty)$ (which satisfies $\left.\xi_{u}(\tau) \in B_{R_{0}}(\varepsilon)\right)$ converges as $t \rightarrow \infty$ to one of these almost-periodic solutions.

3) The pullback attractor $\mathcal{A}_{\phi_{\varepsilon}}(\tau)$ possesses the following description analogous to (5.3):

$$
\mathcal{A}_{\phi_{\varepsilon}}(\tau)=\cup_{z_{0} \in \mathcal{R}_{0}} \mathcal{M}_{\phi_{\varepsilon}, z_{0}}^{+}(\tau), \quad \tau \in \mathbb{R}
$$

where the $C^{1}$-submanifolds $\mathcal{M}_{\phi_{\varepsilon}, z_{0}}^{+}(\tau)$ are the (global) unstable manifolds of the almost-periodic solution $u_{\phi_{\varepsilon}, z_{0}}(t)$ associated with the equilibrium $\xi_{z_{0}} \in \mathcal{R}_{0}$ (which are constructed in the previous section).

4) The pullback attractors $\mathcal{A}_{\phi_{\varepsilon}}$ are uniformly (with respect to $\tau \in \mathbb{R}$ and $\phi_{\varepsilon} \in$ $\left.\mathcal{H}\left(f_{\varepsilon}\right)\right)$ exponential, i.e., there exist positive constant $\alpha$ and a monotonic function $Q$ (which is independent of $\varepsilon, \tau$ and $\phi_{\varepsilon}$ ) such that, for every (bounded) subset $B \subset B_{R_{0}(\varepsilon)}$, we have

$$
\operatorname{dist}_{E^{1}(\Omega)}\left(U_{\phi_{\varepsilon}}(\tau+t, \tau) B, \mathcal{A}_{\phi_{\varepsilon}}(\tau+t)\right) \leq Q\left(\|B\|_{E^{1}(\Omega)}\right) e^{-\alpha t} .
$$

5) The attractors $\mathcal{A}_{\phi_{\varepsilon}}(\tau)$ tend as $\varepsilon \rightarrow 0$ to the limit attractor $\mathcal{A}_{0}$ in the following sense:

$$
\operatorname{dist}_{E^{1}(\Omega)}^{\text {symm }}\left(\mathcal{A}_{\phi_{\varepsilon}}(\tau), \mathcal{A}_{0}\right) \leq C_{\bar{R}}\left[\alpha_{C_{\bar{R}}^{\prime}}(\varepsilon)\right]^{\kappa}
$$

where the function $\alpha_{R}(\varepsilon)$ is the same as in Proposition 2.8, $\bar{R}$ is the same as in Theorem 3.2, the positive constants $\kappa, C_{\bar{R}}$ and $C_{\bar{R}}^{\prime}$ are independent of $\tau, \varepsilon, B$ and $\phi_{\varepsilon}$ and the symbol $\operatorname{dist}_{V}^{\text {symm }}(X, Y)$ denotes the symmetric Hausdorff distance between subsets $X$ and $Y$ of $V$.

Sketch of the proof. We give below only an overview of the proof of Theorem 5.3, (the details can be found in [14], see also [3] and [18]). As usual, this proof is based on the following lemma which allows to reduce the analysis of the global behavior of solutions of (2.3) to the local analysis of equation (2.3) near the equilibria $\xi_{z_{0}} \in \mathcal{R}_{0}$.

Lemma 5.3. Let the assumptions of Theorem 5.1 hold. Then, the following statements are valid:

1) For every neighborhood $V$ of zero in $E^{1}(\Omega)$ and every bounded subset $B \subset$ $E^{1}(\Omega)$, there exist $\varepsilon_{0}=\varepsilon_{0}(B, V)$ and $T=T(B, V)>0$ such that every solution $\xi_{u}(t)$ of equation (2.3) (with $\varepsilon \leq \varepsilon_{0}, \tau \in \mathbb{R}, \phi_{\varepsilon} \in \mathcal{H}\left(f_{\varepsilon}\right)$ ) such that $\xi_{u}(\tau) \in B$ visits the $V$-neighborhood of the set $\mathcal{R}_{0}$ on every time interval of length $T$, i.e., for every $s \geq \tau$, there exists $T_{s}=T_{s}(u) \in[s, s+T]$ and $\xi_{z_{0}} \in \mathcal{R}_{0}$ such that

$$
\xi_{u}\left(T_{s}\right) \in \xi_{z_{0}}+V
$$

2) There exist small neighborhoods $W$ and $W^{\prime}$ of zero in $E^{1}(\Omega), W^{\prime} \subset W$, and positive $\varepsilon_{0}$ such that every solution $\xi_{u}(t), t \geq \tau$ of equation $(2.3)$ (with $\varepsilon \leq \varepsilon_{0}$, $\tau \in \mathbb{R}$ and $\phi_{\varepsilon} \in \mathcal{H}\left(f_{\varepsilon}\right)$ ) satisfies the following condition: if $\xi_{u}(\tau) \in \xi_{z_{0}}+W^{\prime}$ for some $\xi_{z_{0}} \in \mathcal{R}_{0}$ and $\xi_{u}(T) \notin \xi_{z_{0}}+W$ for some $T \geq \tau$, then this trajectory never returns to the $W^{\prime}$-neighborhood of $\xi_{z_{0}}$ :

$$
\xi_{u}(t) \notin \xi_{z_{0}}+W^{\prime}, \quad \forall t \geq T
$$


Sketch of the proof. Assume that the first assertion of the lemma is false. Then, there exist a neighborhood $V_{0}$ of zero, a sequence $T_{n} \rightarrow \infty$ and a sequence of solutions $\xi_{u_{n}}(t):=U_{\phi_{\varepsilon_{n}}}\left(t, \tau_{n}\right) \xi_{n}$ of equation (2.3) such that $\varepsilon_{n} \rightarrow 0$ as $n \rightarrow 0$ and

$$
\xi_{u_{n}}(t) \notin V_{0}+\mathcal{R}_{0}, \forall t \in\left[\tau_{n}, \tau_{n}+T_{n}\right] \text { and } \xi_{u_{n}}\left(\tau_{n}\right) \in B
$$

Let us consider a new sequence of solutions $\xi_{\tilde{u}_{n}}(t):=\xi_{u_{n}}\left(t+\tau_{n}+T_{n} / 2\right)$. Then, (5.9) and (2.21) imply that these solutions are defined on $t \in\left[-T_{n} / 2, T_{n} / 2\right]$ and

$$
\xi_{\tilde{u}}(t) \notin V_{0}+\mathcal{R}_{0} \quad \text { and }\left\|\xi_{\tilde{u}_{n}}(t)\right\|_{E^{1}(\Omega)} \leq C, \forall t \in\left[-T_{n} / 2, T_{n} / 2\right] .
$$

Using Proposition 2.8 and the fact that the limit equation (2.1) possesses a global attractor, we can assume without loss of generality that the sequence $\xi_{\tilde{u}_{n}}(t)$ tends as $n \rightarrow \infty$ to some complete solution $\xi_{\bar{u}} \in \mathcal{K}_{\bar{f}}$ of the limit equation (2.1) (e.g., in the space $L_{l o c}^{\infty}\left(\mathbb{R}, E^{1}(\Omega)\right)$ ). Passing now to the limit $n \rightarrow \infty$ in (5.10), we deduce that $\xi_{\bar{u}}(t) \notin V_{0}+\mathcal{R}$, for all $t \in \mathbb{R}$ which contradicts the fact that (2.1) possesses a global Lyapunov function (see Theorem 5.1). Thus, the first assertion of the lemma is proven.

Analogously, assuming that the second assertion is wrong, we construct a homoclinic structure for the limit equation (2.1) which also contradicts the existence of a global Lyapunov function (see [14] for the details). Lemma 5.3 is proven.

We are now ready to finish the proof of Theorem 5.3. To this end, we fix a neighborhood $V_{0} \subset W^{\prime}$ (where $W^{\prime}$ is the same as in Lemma 5.3) such that the assertions of Theorems 4.1, 4.3, 4.6 be satisfied for all neighborhoods $\xi_{z_{i}}+V_{0}$, $i=1, \cdots, N$. We then fix $B=B_{\bar{R}}$ to be a uniform absorbing set for the processes $U_{\phi_{\varepsilon}}(t, \tau)$ in $E^{1}(\Omega)$ (which exists due to estimate $(2.21)$ ). Finally, we assume that $\varepsilon_{0}>0$ and $T>0$ are such that assertions of Theorems 4.1, 4.3, 4.6 and Lemma 5.3 hold, for every $\varepsilon \leq \varepsilon_{0}$ and every $\xi_{z_{0}} \in \mathcal{R}_{0}$.

Then, the second statement of Lemma 5.3 implies that every solution $\xi_{u}(t)$, $t \geq \tau$ (such that $\varepsilon \leq \varepsilon_{0}$ and $\xi_{u}(\tau) \in B$ ) can leave the neighborhood $V_{0}+\mathcal{R}_{0}$ only a finite number $N_{u} \leq N$ of times and, consequently, due to the first assumption of Lemma 5.3, there exists $\xi_{z_{0}^{+}} \in \mathcal{R}_{0}$ such that $\xi_{u}(t) \in \xi_{z_{0}^{+}}+V_{0}$ for all sufficiently large $t$. Theorem 4.6 and Remark 4.7 now imply that $\xi_{u}(t)$ stabilizes exponentially to $\xi_{u_{\phi_{\varepsilon}, z_{0}^{+}}}(t)$ as $t \rightarrow+\infty$. Analogously, if $\xi_{u} \in \mathcal{K}_{\phi_{\varepsilon}}$ is a complete solution of (2.3), then, due to Lemma 5.3 and the fact that the number of the equilibria is finite, we have $\xi_{u}(t) \in V_{0}+\xi_{z_{0}^{-}}$for all $t$ small enough. Theorem 4.3 implies now that $\xi_{u}(t)$ stabilizes to $\xi_{u_{\phi_{\varepsilon}, z_{0}^{-}}}(t)$ as $t \rightarrow-\infty$. Thus, the first and the second assertions of Theorem 5.3 are verified.

Description (5.5) is an immediate corollary of stabilization (5.4), definition (4.21) of the unstable manifolds and formula (3.24).

The exponential attraction (5.6) is a standard corollary of the facts that every trajectory of (2.1) spends only a finite time $\tilde{T} \leq T \cdot \# \mathcal{R}_{0}$ outside of the neighborhood $V_{0}+\mathcal{R}_{0}$ (due to Lemma 5.3) and that this trajectory is exponentially attracted to $\mathcal{A}_{\phi_{\varepsilon}}(t)$ while staying inside of $V_{0}+\mathcal{R}_{0}$ (due to Theorem 4.6). Moreover, since the time $\tilde{T}$ and the rate of the attraction in Theorem 4.6 are independent of $\varepsilon$, then (5.6) will also be uniform with respect to $\varepsilon$ (see [14] for the details).

Finally, estimate (5.7) is a formal corollary of the uniform exponential attraction (5.6) and Proposition 2.8 (and can be obtained exactly as in Corollary 3.5, see also [3] and [14]). Theorem 5.3 is proven. 
Remark 5.4. We recall that the constructed uniform $\left(\mathcal{A}_{\varepsilon}\right)$ and pullback $\left(\mathcal{A}_{\phi_{\varepsilon}}(\tau)\right)$ attractors of equation (2.3) attract the solutions $u(t)$ whose initial data belong to the large ball $B_{R_{0}(\varepsilon)}$ in $E^{1}(\Omega)$ only (with $\lim _{\varepsilon \rightarrow 0} R_{0}(\varepsilon)=\infty$ ). We however note that, even in the case where we have the global solvability of problem (2.3) for every $\xi^{\tau} \in E^{1}(\Omega)$ and the associated family of processes has the attractor in the whole space $E^{1}(\Omega)$; it does not necessarily coincide with $\mathcal{A}_{\varepsilon}$ and may even diverge to infinity as $\varepsilon \rightarrow 0$. We will give the corresponding examples in Section 7 .

We now formulate several two corollaries of Theorem 5.3.

Corollary 5.5. Let the assumptions of Theorem 5.3 hold. Then the uniform attractors $\mathcal{A}_{\varepsilon}$ of problems (2.3) are upper and lower semicontinuous as $\varepsilon \rightarrow 0$. Moreover, the following estimate holds:

$$
\operatorname{dist}_{E^{1}(\Omega)}^{\text {symm }}\left(\mathcal{A}_{\varepsilon}, \mathcal{A}_{0}\right) \leq C_{\bar{R}}\left[\alpha_{C_{\bar{R}}^{\prime}}(\varepsilon)\right]^{\kappa}
$$

where the right-hand side of (5.11) is the same as in (5.7).

Indeed, estimate (5.11) is an immediate corollary of (5.7) and (3.25).

Corollary 5.6. Let the assumptions of Theorem 5.3 and Lemma 1.6 hold (e.g., let $f_{\varepsilon}$ be periodic with respect to $t$ ). Then, estimate (5.7) can be improved as follows:

$$
\operatorname{dist}_{E^{1}(\Omega)}^{\text {symm }}\left(\mathcal{A}_{\phi_{\varepsilon}}(\tau), \mathcal{A}_{0}\right) \leq C_{\bar{R}}^{\prime \prime} \varepsilon^{\kappa}
$$

where $\bar{R}$ and $\kappa>0$ are the same as in (5.7) and the positive constant $C_{\bar{R}}^{\prime \prime}$ is independent of independent of $\tau, \varepsilon$ and $\phi_{\varepsilon}$.

Indeed, inserting estimate (1.30) into the right-hand side of (5.7), we derive (5.12).

Remark 5.7. It is worth to note that, under the assumptions of Theorem 5.3, we have a simpler relation between the uniform and pullback attractors, namely,

$$
\mathcal{A}_{\varepsilon}=\left[\cup_{t \in \mathbb{R}} \mathcal{A}_{\phi_{\varepsilon}}(t)\right]_{E^{1}(\Omega)}, \quad \text { for every fixed } \phi_{\varepsilon} \in \mathcal{H}\left(f_{\varepsilon}\right)
$$

Indeed, description (5.13) follows from the uniform attraction (5.6) and the alternative definition of the uniform attractor, see Remark 3.3.

It is also worth to note that, under the assumptions of Theorem 5.3, the pullback attractors $\mathcal{A}_{\phi_{\varepsilon}}(t)$ are almost-periodic with respect to $t$ as the set-valued functions $t \rightarrow \mathcal{A}_{\phi_{\varepsilon}}(t)$ for every $\phi_{\varepsilon} \in \mathcal{H}\left(f_{\varepsilon}\right)$, see [14] for details.

\section{$\S 6$ The subordinated oscillations.}

In this section, we study the case of the so-called subordinated oscillations where we have the global existence of solutions and uniform (with respect to $\varepsilon \rightarrow 0$ ) dissipativity of system (2.3) not only for $\xi^{\tau} \in B_{R_{0}(\varepsilon)}$, but for every $\xi^{\tau} \in E^{1}(\Omega)$ (and even, for every $\xi^{\tau} \in E(\Omega)$ ).

We first recall that in the previous sections we imposed the dissipativity and growth assumptions (see (1.8)) to the averaged function $\bar{f}$ only, so, if we want to have the global solvability of problems (2.3) for arbitrary $\xi^{\tau} \in E^{1}(\Omega)$, we need to impose some assumptions to the functions $\phi_{\varepsilon}(u, t)$ for positive $\varepsilon$. It seems natural to require, analogously to the case of autonomous equation (2.1), the nonlinearity 
$f_{\varepsilon}(u, t)$ to satisfy conditions (1.8) uniformly with respect to $t$ and $\varepsilon$. We however note that, in the non-autonomous case, the sole assumption (1.8) is not sufficient to obtain the dissipative estimate for the solutions of (2.3) (see e.g. Example 7.4 below). The standard additional assumption, see e.g. [8], [9], which guarantees the dissipativity of the non-autonomous equation (2.3), is the following one:

$$
\partial_{t} \phi_{\varepsilon}(u, t) \leq \delta \phi_{\varepsilon}(u, t) \cdot u+C_{\delta}, \quad \forall t, u \in \mathbb{R}
$$

where $\delta=\delta(\gamma)$ is a sufficiently small positive number. We however note that the function $\phi_{\varepsilon}(u, t)$ contains the rapidly oscillating term $t / \varepsilon$, so the derivative $\partial_{t} \phi_{\varepsilon}$ is of order $1 / \varepsilon$ as $\varepsilon \rightarrow 0$ and, consequently, estimate (6.1) cannot be uniform with respect to $\varepsilon$. Thus, using (6.1), we cannot obtain uniform (with respect to $\varepsilon$ ) bounds for the corresponding attractors.

This is why, instead of (6.1), we use below the following (in a sense, more restrictive) assumption that

$$
\left|f_{\varepsilon}(u, t)-\bar{f}(u)\right|^{2} \leq \delta \bar{f}(u) . u+C_{\delta}, \quad \forall t, u \in \mathbb{R}, \quad \varepsilon \geq 0
$$

where $\delta$ and $C_{\delta}$ is independent of $\varepsilon$ and $t$ and $\delta=\delta(\gamma)$ is small enough. In particular, (6.2) implies that the leading part of the non-linearity $f_{\varepsilon}(u, t)$ is autonomous (which justifies the title "subordinated oscillations" of this section. We start from the following theorem, which gives the uniform (with respect to $\varepsilon$ ) dissipative estimate in the space $E(\Omega)$ for the solutions of $(2.3)$.

Theorem 6.1. Let the functions $f(\varepsilon, u, z)$ satisfy the assumptions of Lemma 1.1. Assume, in addition that estimate (6.2) holds, where the average $\bar{f}$ satisfies assumptions (1.8) and that the following growth restriction holds:

$$
\left|\partial_{u}^{2} f_{\varepsilon}(u, t)\right| \leq C(1+|u|), \quad \forall t, u \in \mathbb{R}
$$

where $C$ is independent of $\varepsilon$. Then, for every $\varepsilon \geq 0, \tau \in \mathbb{R}, \phi_{\varepsilon} \in \mathcal{H}\left(f_{\varepsilon}\right)$ and $\xi^{\tau} \in E(\Omega)$, equation (2.3) possesses a unique solution $\xi_{u}(t) \in E(\Omega)$ for every $t \geq \tau$ and the following estimate holds:

$$
\left\|\xi_{u}(t)\right\|_{E(\Omega)} \leq Q\left(\left\|\xi^{\tau}\right\|_{E(\Omega)}\right) e^{-\alpha(t-\tau)}+Q\left(\|g\|_{L^{2}(\Omega)}\right)
$$

where the positive constant $\alpha$ and the monotonic function $Q$ are independent of $\varepsilon$, $\tau, \phi_{\varepsilon} \in \mathcal{H}\left(f_{\varepsilon}\right)$ and $\xi^{\tau} \in E(\Omega)$.

Proof. Although the assertion of the theorem is more or less standard, we give below the derivation of (6.4), in order to show that it is indeed uniform with respect to $\varepsilon$. For simplicity, we consider below only the case $\phi_{\varepsilon}=f_{\varepsilon}$ and $\tau=0$ (the general case is analogous due to Lemma 1.3). Moreover, we only give the formal derivation of estimate (6.4) which can be easily justified using, e.g., the Galerkin approximations method (we recall that assumption (6.3) guarantees the uniqueness of a solution to (2.3) in the three-dimensional case, see [3]). To this end, following the standard procedure, see e.g. [3] or [29], we multiply equation (2.3) by $\partial_{t} u+\beta u$, for some positive $\beta$, and integrate over $\Omega$. Then, after the integration by parts, we have

$$
\partial_{t}\left[\left\|\xi_{u}(t)\right\|_{E(\Omega)}^{2}+2\|F(u(t))\|_{L^{1}(\Omega)}+\lambda_{0}\|u(t)\|_{L^{2}(\Omega)}^{2}+2 \beta\left(u(t), \partial_{t} u(t)\right)\right]+
$$

$$
\begin{array}{r}
+2(\gamma-\beta)\left\|\partial_{t} u(t)\right\|_{L^{2}(\Omega)}^{2}+2 \beta\left\|\nabla_{x} u(t)\right\|_{L^{2}(\Omega)}^{2}+2 \lambda_{0}\|u(t)\|_{L^{2}(\Omega)}^{2}+2 \beta(\bar{f}(u(t)), u(t))= \\
=2\left(g, \partial_{t} u(t)-\beta u(t)\right)-2\left(\tilde{f}_{\varepsilon}(u(t), t), \partial_{t} u(t)+\beta u(t)\right)
\end{array}
$$


where $F(v):=\int_{0}^{v} \bar{f}(u) d u$ and $\tilde{f}_{\varepsilon}(u, t):=f_{\varepsilon}(u, t)-\bar{f}(u)$. We recall that dissipativity assumption (1.8)(3) implies that

$$
F(v) \leq f(v) \cdot v+C, \quad f(v) \cdot v \geq-C_{\mu}-\mu|v|^{2}, \quad \text { and } \quad F(v) \geq-C_{\mu}-\mu|v|^{2}
$$

where $\mu>0$ can be arbitrarily small and the constants $C$ and $C_{\mu}$ are independent of $v \in \mathbb{R}$. Using now estimates (6.2), (6.6) and Cauchy-Schwarz inequality, we derive from (6.5) that there exists sufficiently small (but independent of $\varepsilon$ ) positive constants $\beta=\beta(\gamma), \delta=\delta(\gamma)$ (which is the same as in assumption (6.2)) and $\alpha=\alpha(\gamma)$ such that

$$
\begin{aligned}
\partial_{t}\left[\left\|\xi_{u}(t)\right\|_{E(\Omega)}^{2}+\right. & \left.2\|F(u(t))\|_{L^{1}(\Omega)}+\lambda_{0}\|u(t)\|_{L^{2}(\Omega)}^{2}+2 \beta\left(u(t), \partial_{t} u(t)\right)\right]+ \\
+ & \alpha\left[\left\|\xi_{u}(t)\right\|_{E(\Omega)}^{2}+2\|F(u(t))\|_{L^{1}(\Omega)}+\right. \\
& \left.+\lambda_{0}\|u(t)\|_{L^{2}(\Omega)}^{2}+2 \beta\left(u(t), \partial_{t} u(t)\right)\right] \leq C\left(1+\|g\|_{L^{2}(\Omega)}^{2}\right) .
\end{aligned}
$$

Applying the Gronwall's inequality to this relation, we derive estimate (6.4) and Theorem 6.1 is proven.

Thus, under the assumptions of Theorem 6.1, equations (2.3) define a family of dynamical processes which are defined globally on $E(\Omega)$ :

$$
U_{\phi_{\varepsilon}}(t, \tau): E(\Omega) \rightarrow E(\Omega), \quad \phi_{\varepsilon} \subset \mathcal{H}\left(f_{\varepsilon}\right), \quad \tau \in \mathbb{R}, t \geq \tau .
$$

The main result of this section is the following theorem which establishes the existence of a uniform attractor for (6.8) and verifies that, for small $\varepsilon$, this attractor coincides with the one constructed in Theorem 3.2 starting from the ball $B_{R_{0}(\varepsilon)}$ of the space $E^{1}(\Omega)$.

Theorem 6.2. Let the assumptions of Theorem 6.1 hold. Then, for every $\varepsilon>0$, family (6.8) possesses a uniform attractor $\mathcal{A}_{\varepsilon}$ which is compact in $E(\Omega)$ and is uniformly bounded with respect to $\varepsilon$ :

$$
\left\|\mathcal{A}_{\varepsilon}\right\|_{E(\Omega)} \leq C
$$

Moreover, there exists small positive $\varepsilon_{0}$ such that, for every $\varepsilon \leq \varepsilon_{0}$, the attractors $\mathcal{A}_{\varepsilon}$ are compact in $E^{1}(\Omega)$ and

$$
\left\|\mathcal{A}_{\varepsilon}\right\|_{E^{1}(\Omega)} \leq C_{1}
$$

where the constant $C_{1}$ is independent of $\varepsilon$.

Proof. We first consider the case of small $\varepsilon$, where we have regularity (6.10) of the attractor. We also recall that, in the subcritical case, where the growth rate of $f_{\varepsilon}$ is strictly less than cubic (see assumptions (1.21)) this regularity can be obtained using, e.g., the bootstrap arguments, exactly as in the autonomous case, see [3]. So, we mainly consider the critical case of a cubic rate of growth. In this case, the derivation of dissipative estimate $(2.2)$ in the $E^{1}(\Omega)$-norm in the autonomous case (see [3]) essentially uses the so-called dissipation integral which equals infinity in the non-autonomous case. Therefore, the methods of [3] cannot be directly applied in order to obtain regularity (6.10). Nevertheless, there is a possibility to adapt 
these methods to equation (2.3) with small $\varepsilon>0$. Since we are mainly interested in the limit $\varepsilon \rightarrow 0$ this is enough for our purposes (see the recent paper [35] for the case of damped wave equations with general nonautonomous external forces).

We give below only the proof of the $E^{\alpha}(\Omega)$-regularity of the attractor $\mathcal{A}_{\varepsilon}$ for some positive $\alpha$ which is the most difficult part of the derivation of regularity (6.10) in the critical cubic rate of growth leaving the proof of $E^{1}$-regularity to the reader (since the cubic rate of growth is subcritical with respect to the $E^{\alpha}(\Omega)$-norm, then the bootstrap arguments work starting with the $E^{\alpha}(\Omega)$-energy and allow to deduce estimate (6.10) exactly as in the autonomous case, see [3] and [29] for the details). Thus, due to the standard theorem on the existence of a global attractor, see the proof of Theorem 3.2, we only need to prove the following proposition which gives the uniform (with respect to $\varepsilon$ ) attracting set in $E^{\alpha}(\Omega)$.

Proposition 6.3. Let the assumptions of Theorem 6.2 hold. Then, there exist $\varepsilon_{0}>0, \alpha>0$ and a sufficiently large ball $B\left(E^{\alpha}\right)$ of the space $E^{\alpha}(\Omega)$ such that, for every $\varepsilon \in\left(0, \varepsilon_{0}\right]$ and every bounded subset $B \subset E(\Omega)$, we have

$$
\operatorname{dist}_{E(\Omega)}\left(U_{\phi_{\varepsilon}}(t+\tau, \tau) B, B\left(E^{\alpha}\right)\right) \leq Q\left(\|B\|_{E(\Omega)}\right) e^{-\beta t}
$$

where the positive constant $\beta$ and the monotonic function $Q$ are independent of $t$, $\tau, \varepsilon$ and $\phi_{\varepsilon}$.

Proof. As before, we consider below only the case $\phi_{\varepsilon}=f_{\varepsilon}$ and $\tau=0$ (the general case is analogous due to Lemma 1.3). Moreover, due to estimate (6.4), we may prove (6.11) only for the ball $B=B_{R}$ of a sufficiently large radius $R$ in $E(\Omega)$.

In order to handle the rapid oscillations in time in equation (2.3), it is convenient to introduce the auxiliary function $w(t)=w_{u}(t)$ which solves the following equation

$$
\partial_{t}^{2} w+\gamma \partial_{t} w-\Delta_{x} w+\lambda_{0} w=h_{u}(t):=-\tilde{f}_{\varepsilon}(u(t), t),\left.\quad \xi_{w}\right|_{t=0}=0
$$

Then, due to assumptions (6.2) and (6.3), the function $\tilde{f}_{\varepsilon}$ satisfies condition (1.21) (with $\delta=1$ ). Consequently, thanks to Lemma 1.5, estimate (6.4) and Proposition A.2, we have

$$
\left\|\xi_{w}(t)\right\|_{E^{-1}(\Omega)} \leq \alpha(\varepsilon), \forall t \geq 0, \xi^{0} \in B_{R}
$$

where the monotonic function $\alpha$ tends to zero as $\varepsilon \rightarrow 0$. Moreover, it follows from (6.2), (6.3), (6.4) and Hölder inequality that

$$
\left\|\nabla_{x} h_{u}(t)\right\|_{L^{3 / 2}(\Omega)}+\left\|h_{u}(t)\right\|_{L^{3}(\Omega)} \leq C
$$

Applying the appropriate interpolation inequality to (6.14), we derive that there exists positive $\alpha$, such that

$$
\left\|h_{u}(t)\right\|_{H^{2 \alpha}(\Omega)} \leq C_{1}
$$

Applying Proposition A.1 to equation (6.12) and using (6.13) together with the interpolation inequality, we finally derive that

$$
\left\|\xi_{w}(t)\right\|_{E^{\alpha}(\Omega)} \underset{32}{\leq} \tilde{\alpha}(\varepsilon), \quad t \in \mathbb{R}_{+}
$$


where the monotonic function $\tilde{\alpha}$ is independent of $t$ and $\xi^{0} \in B_{R}$ and tends to zero as $\varepsilon \rightarrow 0$.

We now set $v(t):=u(t)-w(t)$, where $u(t)$ solves (2.3). Then, this function satisfies the equation

$$
\partial_{t}^{2} v+\gamma \partial_{t} v-\Delta_{x} v+\lambda_{0} v+\bar{f}(v+w(t))=g,\left.\quad \xi_{v}\right|_{t=0}=\left.\xi_{u}\right|_{t=0}
$$

Thus, instead of equation (2.3), we will prove the existence of an exponentially attracting set in $E^{\alpha}(\Omega)$ for equation (6.17). To this end, we split (following [3]) the solution $v(t)$ as follows $v(t)=v_{0}(t)+\theta(t)$, where $v_{0}(t)$ is a solution of the following autonomous equation:

$$
\partial_{t}^{2} v_{0}+\gamma \partial_{t} v_{0}-\Delta_{x} v_{0}+\lambda v_{0}+\bar{f}\left(v_{0}\right)+L v_{0}=0,\left.\quad \xi_{v_{0}}\right|_{t=0}=\left.\xi_{u}\right|_{t=0}
$$

where $L$ is a sufficiently large positive number and the remainder $\theta(t)$ satisfies the equation

$$
\partial_{t}^{2} \theta+\gamma \partial_{t} \theta-\Delta_{x} \theta+\lambda_{0} \theta+\left[\bar{f}\left(\theta+v_{0}+w\right)-\bar{f}\left(v_{0}\right)\right]=g+L v_{0}(t),\left.\xi_{\theta}\right|_{t=0}=0
$$

where we may assume without loss of generality that $\bar{f}(0)=0$. Then, arguing analogously to the proof of Theorem 6.1 , we derive that the solution $v_{0}(t)$ decays exponentially if $L=L(\bar{f})$ is large enough:

$$
\left\|\xi_{v_{0}}(t)\right\|_{E(\Omega)} \leq Q\left(\left\|\xi_{u}(0)\right\|_{E(\Omega)}\right) e^{-\beta t}, \quad t \geq 0
$$

where the positive constant $\beta$ and the monotonic function $Q$ is independent of $\xi^{0}$ and $t$, see [3] for the details. Thus, in order to finish the proof of Proposition 6.3, there only remains to verify that the solution $\theta(t)$ is uniformly bounded in $E^{\alpha}(\Omega)$. To this end, we need the following lemma which plays the role of a "dissipation integral" in the case of small positive $\varepsilon$.

Lemma 6.4. Let the above assumptions hold. Then, the following estimate is valid for the solution $v(t)$ of equation (6.17):

$$
\int_{0}^{T}\left\|\partial_{t} v(t)\right\|_{L^{2}(\Omega)}^{2} d t \leq C(T+1) \tilde{\alpha}(\varepsilon), \quad T \in \mathbb{R}_{+}
$$

where the function $\tilde{\alpha}$ is the same as in (6.16) and the constant $C$ is independent of $\varepsilon, \xi^{0} \in B_{R}$ and $T$.

Indeed, estimate (6.21) can be obtained in a standard way multiplying equation (6.17) by $\partial_{t} v(t)$, integrating over $[0, T] \times \Omega$ and using estimates (6.4) and (6.16).

Let us now differentiate equation (6.19) with respect to $t$ and denote $W(t):=$ $\partial_{t} \theta(t)$. Then, we have

$$
\partial_{t}^{2} W+\gamma \partial_{t} W-\Delta_{x} W+\lambda_{0} W=-\bar{f}^{\prime}\left(v_{0}\right)\left(W+\partial_{t} w\right)+
$$$$
\left(\bar{f}^{\prime}\left(v_{0}+\theta+w\right)-\bar{f}^{\prime}\left(v_{0}\right)\right) \partial_{t} u(t)+L \partial_{t} v_{0}(t):=h_{1}(t)+h_{2}(t)+h_{3}(t), \quad \xi_{W}(0)=(0, g) \text {. }
$$ 
Multiplying equation (6.22) by $\left(-\Delta_{x}+\lambda_{0}\right)_{N}^{\alpha-1}\left(\partial_{t} W+\beta W\right)$, where $\alpha>0$ is the same as in estimate (6.16) (without loss of generality, we may assume that $\alpha<1 / 2$ ) and $\beta$ is a sufficiently small positive number, we derive that

$$
\begin{gathered}
\partial_{t}\left[\left\|\xi_{W}(t)\right\|_{E^{\alpha-1}}^{2}+2 \beta\left(\partial_{t} W(t), W(t)\right)_{H^{\alpha-1}}\right]+2(\gamma-\beta)\left\|\partial_{t} W(t)\right\|_{H^{\alpha-1}}^{2}+ \\
+2 \beta\|W(t)\|_{H^{\alpha}}^{2}=2\left(h_{1}(t),\left(-\Delta_{x}+\lambda_{0}\right)_{N}^{\alpha-1}\left(\partial_{t} W(t)+\beta W(t)\right)\right)+ \\
+2\left(h_{2}(t),\left(-\Delta_{x}+\lambda_{0}\right)_{N}^{\alpha-1}\left(\partial_{t} W(t)+\beta W(t)\right)\right)+ \\
+2\left(h_{3}(t),\left(-\Delta_{x}+\lambda_{0}\right)_{N}^{\alpha-1}\left(\partial_{t} W(t)+\beta W(t)\right)\right) .
\end{gathered}
$$

In order to estimate the right-hand side of (6.23), we need the following standard inequalities:

$$
\left\{\begin{array}{l}
\left\|u_{1} \cdot\left(-\Delta_{x}+\lambda_{0}\right)_{N}^{\alpha-1} u_{2}\right\|_{L^{3}(\Omega)} \leq C\left\|u_{1}\right\|_{H^{1+\alpha}}\left\|u_{2}\right\|_{H^{\alpha-1}} \\
\left\|u_{3} \cdot\left(-\Delta_{x}+\lambda_{0}\right)_{N}^{\alpha-1} u_{2}\right\|_{L^{3 / 2}(\Omega)} \leq C\left\|u_{3}\right\|_{H^{\alpha}}\left\|u_{2}\right\|_{H^{\alpha-1}}
\end{array}\right.
$$

which hold for every $u_{1} \in H^{\alpha+1}(\Omega), u_{2} \in H^{\alpha-1}(\Omega)$ and $u_{3} \in H^{\alpha}(\Omega)$ and every $0 \leq \alpha<1 / 2$ (indeed, these estimate can be easily verified using (1.13), Sobolev's embedding theorem and the appropriate Hölder's inequality, see [35]).

Applying now Hölder's inequality to the first term in the right-hand side of (6.23) and using estimate $(6.24)(2)$, we have

$$
\begin{aligned}
& \left|\left(h_{1},\left(-\Delta_{x}+\lambda_{0}\right)_{N}^{\alpha-1}\left(\partial_{t} W+\beta W\right)\right)\right| \leq \\
& \quad \leq C\left\|\bar{f}\left(v_{0}(t)\right)\right\|_{L^{3}(\Omega)}\left\|W+\beta \partial_{t} w\right\|_{H^{\alpha}}\left\|\partial_{t} W+\beta W\right\|_{H^{\alpha-1}(\Omega)} \leq \\
& \quad \leq C_{1}\left\|\bar{f}\left(v_{0}(t)\right)\right\|_{L^{3}(\Omega)}\left(\left\|\xi_{W}(t)\right\|_{E^{\alpha-1}}^{2}+\left\|\xi_{w}(t)\right\|_{E^{\alpha}}^{2}\right)
\end{aligned}
$$

In order to estimate the second term, we first note that, expressing the term $\Delta_{x} \theta$ from equation (6.19) and using the elliptic regularity theorem for the Laplacian and the fact that the $E(\Omega)$-norm of $\xi_{\theta}$ and $\xi_{v_{0}}$ are uniformly bounded, we derive that

$$
\|\theta(t)\|_{H^{1+\alpha}} \leq C_{2}\left(\left\|\xi_{W}(t)\right\|_{E^{\alpha-1}(\Omega)}+1\right) .
$$

Applying Hölder's inequality to the second term in the right-hand side of (6.23) and using (6.24)(1), (6.26) and the growth restriction (6.3), we have

$$
\begin{aligned}
& \left|\left(h_{2},\left(-\Delta_{x}+\lambda_{0}\right)_{N}^{\alpha-1}\left(\partial_{t} W+\beta W\right)\right)\right| \leq C^{\prime}\left(\|u\|_{L^{6}(\Omega)}+\left\|v_{0}\right\|_{L^{6}(\Omega)}\right) \times \\
& \times\left\|\partial_{t} u\right\|_{L^{2}(\Omega)}\|\theta+w\|_{H^{1+\alpha}}\left\|\partial_{t} W+\beta W\right\|_{H^{\alpha-1}} \leq \\
& \quad \leq C^{\prime \prime}\left\|\partial_{t} u(t)\right\|_{L^{2}(\Omega)}\left(\left\|\xi_{W}(t)\right\|_{E^{\alpha-1}}^{2}+\left\|\xi_{w}(t)\right\|_{E^{\alpha}}^{2}+1\right) .
\end{aligned}
$$

Inserting estimates (6.25) and (6.27) into the right-hand of (6.23) and using that the $E^{\alpha}$-norm of $\xi_{w}$ is uniformly bounded (thanks to $(6.16)$, we finally derive that

$$
\partial_{t} E_{W}(t)+c(t) E_{W}(t) \leq C^{\prime \prime \prime}
$$

where $E_{W}(t):=\left\|\xi_{W}(t)\right\|_{E^{\alpha-1}}^{2}+2 \beta \lambda_{0}\|W(t)\|_{H^{\alpha-1}}^{2}+2 \beta\left(\partial_{t} W(t), W(t)\right)_{H^{\alpha-1}}$ and the function $c(t)$ has the following form:

$$
c(t)=\gamma_{0}-C^{\prime \prime \prime}\left(\left\|\bar{f}\left(v_{0}(t)\right)\right\|_{L^{3}(\Omega)}+\left\|\partial_{t} u(t)\right\|_{L^{2}(\Omega)}\right)
$$


for some positive constant $\gamma_{0}$. It only remains to note that estimates (6.16), (6.20) and (6.21) imply that there exists small positive $\varepsilon_{0}$ such that, for every $\varepsilon \leq \varepsilon_{0}$, we have

$$
\int_{0}^{T} c(t) d t \geq \gamma_{0} T / 2-C_{3}, \quad \forall T \in \mathbb{R}_{+}
$$

where the constant $C_{3}$ is independent of $T$ and $\varepsilon$. Thus, Gronwall's inequality applied to (6.28) gives

$$
\left\|\xi_{W}(t)\right\|_{E^{\alpha-1}(\Omega)} \leq C_{4}, \forall t \in \mathbb{R}_{+}
$$

and, returning back to the variable $\theta(t)$ (using (6.26)), we prove that

$$
\left\|\xi_{\theta}(t)\right\|_{E^{\alpha}(\Omega)} \leq C_{5}, \quad \forall t \in \mathbb{R}_{+}
$$

where the constant $C_{5}$ is independent of $\varepsilon \leq \varepsilon_{0}, t$ and $\xi^{0} \in B_{R}$. Estimates (6.16), (6.20) and (6.32) give (6.11) and finish the proof of Proposition 6.3.

Therefore, we have proven that, for $\varepsilon \leq \varepsilon_{0}$, family (6.8) of the dynamical processes associated with equation (2.3) possesses a uniform attractor $\mathcal{A}_{\varepsilon}$ which is uniformly (with respect to $\varepsilon$ ) bounded in the space $E^{\alpha}(\Omega)$ for some positive exponent $\alpha<1 / 2$. Since the cubical rate of growth of the nonlinearity is subcritical with respect to the $E^{\alpha}(\Omega)$-norm, then, starting with this $E^{\alpha}(\Omega)$-estimate and using the bootstrap arguments (exactly as in the subcritical case, see e.g. [3]), we obtain the required estimate (6.10). Thus, the second part of Theorem 6.2 is proven.

Let us now consider the case of an arbitrary (not necessarily small) $\varepsilon>0$. In this case, 'dissipation integral' (6.21) is not necessarily small and we cannot obtain estimate (6.30). Therefore, instead of estimate (6.32), we have only that

$$
\left\|\xi_{\theta}(t)\right\|_{E^{\alpha}(\Omega)} \leq C e^{K t},
$$

for some positive constant $K$. Although estimate (6.33) is not strong enough in order to construct a bounded attracting set in $E^{\alpha}(\Omega)$ for positive $\alpha$, it obviously (since $E(\Omega) \subset \subset E^{\alpha}(\Omega)$ ) implies that

$$
\mathbb{K}_{E(\Omega)}\left(U_{\phi_{\varepsilon}}(t+\tau, \tau) B\right) \leq C e^{-\beta t}
$$

where $\mathbb{K}_{V}(X)$ is a Kuratowski measure of noncompactness of the set $X$ in the space $V$ (i.e., the infimum over all $\mu>0$ for which the set $X$ possesses the finite covering by $\mu$-balls of $V$ ) and the positive constants $C$ and $\beta$ are independent of $\phi_{\varepsilon}, t$ and $\tau$. This estimate implies the analogous estimate for the extended semigroup $\mathbb{S}_{t}^{\varepsilon}$ on $E(\Omega) \times \mathcal{H}\left(f_{\varepsilon}\right)$ associated with family $(6.8)$ :

$$
\mathbb{K}_{E(\Omega) \times \mathcal{H}\left(f_{\varepsilon}\right)}\left(\mathbb{S}_{t}^{\varepsilon}\left(B \times \mathcal{H}\left(f_{\varepsilon}\right)\right)\right) \leq C e^{-\beta t}
$$

which is enough to conclude that this semigroup possesses a global attractor in $E(\Omega) \times \mathcal{H}\left(f_{\varepsilon}\right)$, see e.g. [19]. Thus, Theorem 6.2 is proven.

Remark 6.5. Arguing as in the proof of Theorem 6.2, we may prove that, in the case $\varepsilon \leq \varepsilon_{0}$, equation (2.3) possesses a global solution $\xi_{u}(t) \in E^{1}(\Omega)$, for every $\xi^{\tau} \in E^{1}(\Omega)$ which satisfies the following dissipative estimate:

$$
\left\|\xi_{u}(t)\right\|_{E^{1}(\Omega)} \leq Q\left(\left\|\xi^{\tau}\right\|_{E^{1}(\Omega)}\right) e^{-\alpha(t-\tau)}+Q\left(\|g\|_{L^{2}(\Omega)}\right)
$$

where the positive constant $\alpha$ and the monotonic function $Q$ are independent of $\varepsilon$. 
Corollary 6.6. Let the assumptions of Theorem 6.1 hold. Then, there exists $\varepsilon_{0}^{\prime}>0$ such that, for any $\varepsilon \in\left(0, \varepsilon_{0}^{\prime}\right)$ the uniform attractor of the processes (3.1) (defined on the phase space $\left.B_{R_{0}(\varepsilon)} \subset E^{1}(\Omega)\right)$ which is constructed in Theorem 3.2 coincides with the uniform attractor of processes (6.8) (defined on $E(\Omega)$ ) which exists due to Theorem 6.2. In particular, if, in addition, assumptions of Theorem 5.3 are satisfied, then the pullback attractors $\mathcal{A}_{\phi_{\varepsilon}}(\tau)$ of processes (6.8) are regular (i.e., satisfy the properties, formulated in Theorem 5.3).

Remark 6.7. We note that the assertion of Corollary 6.6 may be false if the conditions of Theorem 6.1 are violated, even in the case where equation (2.3) has globally defined and bounded solutions, for every $\xi^{\tau} \in E(\Omega)$, and the corresponding uniform attractor is bounded in $E^{1}(\Omega)$, for every fixed $\varepsilon>0$. We give the corresponding example in the next section.

Remark 6.8. To conclude, we recall that, according to Corollary 6.6 and Theorem 5.1 , the pullback attractors $\mathcal{A}_{\phi_{\varepsilon}}(\tau)$ attract exponentially only bounded in $E^{1}(\Omega)$ subsets, see estimate (5.6). Nevertheless, it is not difficult to deduce from (5.6) and (6.11), using the so-called transitivity of the exponential attraction, see [15], that every bounded in $E(\Omega)$ subsets are also attracted exponentially to these attractors, i.e., for every bounded $B \subset E(\Omega)$, we have

$$
\operatorname{dist}_{E(\Omega)}\left(U_{\phi_{\varepsilon}}(t+\tau, \tau) B, \mathcal{A}_{\phi_{\varepsilon}}(t+\tau)\right) \leq Q\left(\|B\|_{E(\Omega)}\right) e^{-\alpha t}
$$

where the constant $\alpha>0$ and the monotonic function $Q$ are independent of $\varepsilon \leq \varepsilon_{0}^{\prime}$, $\phi_{\varepsilon} \in \mathcal{H}\left(f_{\varepsilon}\right), \tau \in \mathbb{R}$ and $t \geq 0$.

\section{$\S 7$ EXAMPLES AND CONCLUDING REMARKS.}

In this concluding section we illustrate the results obtained above by several concrete examples of equations of the form (0.1). We start with the most natural example of the subordinated oscillations.

Example 7.1. Let us consider the following semilinear hyperbolic problem in a bounded smooth domain $\Omega \subset \mathbb{R}^{3}$ :

$$
\partial_{t}^{2} u+\gamma \partial_{t} u-\Delta_{x} u+u^{3}-a\left(\frac{t}{\varepsilon}\right) u=g,\left.\quad \xi_{u}\right|_{t=\tau}=\xi^{\tau},\left.\quad \partial_{n} u\right|_{\partial \Omega}=0
$$

where $a(z)$ is an almost-periodic (in the sense of Bohr) real-valued function. Then, the averaged equation for (7.1), obviously, has the following form:

$$
\partial_{t}^{2} \bar{u}+\gamma \partial_{t} \bar{u}-\Delta_{x} \bar{u}+\bar{u}^{3}-\bar{a} \bar{u}=g,\left.\quad \xi_{\bar{u}}\right|_{t=\tau}=\xi^{\tau},\left.\quad \partial_{n} \bar{u}\right|_{\partial \Omega}=0
$$

where $\bar{a}:=\mathbb{M}(a)$ is the average of the almost-periodic function $a(z)$.

It is not difficult to verify that equation (7.1) satisfies all of the assumptions of Theorem 6.2 and, consequently, for every $\varepsilon \geq 0$, equation (7.1) possesses a uniform attractor $\mathcal{A}_{\varepsilon}$ in the whole phase space $E(\Omega)$ which is uniformly (with respect to $\varepsilon$ ) bounded in it. Furthermore, according to the second part of Theorem 6.2 , these attractors $\mathcal{A}_{\varepsilon}$ are uniformly bounded in $E^{1}(\Omega)$ if $\varepsilon \leq \varepsilon_{0}$ is small enough (we recall that the nonlinearity in equation (7.1) has a critical cubic growth where the higher regularity of the attractors is rather delicate problem especially in the non-autonomous case in absence of the dissipation integral and, to the best of our 
knowledge, the $E^{1}(\Omega)$-regularity of the attractors of equation (7.1) (even for small positive $\varepsilon$ ) has not been known before).

Moreover, thanks to Theorem 3.2 and Corollary 6.6, the uniform attractors $\mathcal{A}_{\varepsilon}$ tend as $\varepsilon \rightarrow 0$ to the global attractor $\mathcal{A}_{0}$ in the space $E^{1}(\Omega)$ (in the sense of the upper semicontinuity). Finally, under the additional generic assumption that all of the equilibria of the averaged problem (7.2) are hyperbolic, we have (due to Theorem 5.3) also the lower semicontinuity of these uniform attractors as $\varepsilon \rightarrow 0$ and the associated pullback attractor $\mathcal{A}_{f_{\varepsilon}}(\tau)$ is regular (and satisfies assertions 1) -5 ) of Theorem 5.3) if $\varepsilon \leq \varepsilon_{0}$ is small enough.

In the next example we apply the results of previous sections to the autonomous equation with supercritical nonlinearity.

Example 7.2. Let us consider the following semilinear hyperbolic problem in a bounded domain $\Omega \subset \mathbb{R}^{3}$ :

$$
\partial_{t}^{2} u+\gamma \partial_{t} u-\Delta_{x} u+\varepsilon u|u|^{p}+u^{3}-\bar{a} u=g,\left.\quad \xi_{u}\right|_{t=0}=\xi^{0},\left.\quad \partial_{n} u\right|_{\partial \Omega}=0
$$

where $\bar{a} \in \mathbb{R}$ is an arbitrary and the exponent $p>2$. In this case the nonlinearity has the supercritical rate of growth and, a priori, we only have the global existence (without uniqueness) of weak energy solutions of equation (7.3). Nevertheless, since the nonlinearity of equation (7.3) satisfies the assumptions of Section 2, then, due to Corollary 2.6, this equation possesses a (unique) global strong solution $\xi_{u}(t) \in$ $E^{1}(\Omega)$ if $\varepsilon \leq \varepsilon_{0}$ and the initial $E^{1}(\Omega)$-energy is not very large, i.e. $\left\|\xi^{0}\right\|_{E^{1}(\Omega)} \leq$ $R_{0}(\varepsilon)$ where the monotonic function $R_{0}(\varepsilon)$ tends to $+\infty$ as $\varepsilon \rightarrow 0$. Moreover, the semigroup $S_{t}^{\varepsilon}$ generated by this equation on the ball $B_{R_{0}(\varepsilon)}$ of the space $E^{1}(\Omega)$ possesses (thanks to Theorem 3.2) a global attractor $\mathcal{A}_{\varepsilon}$ which is uniformly bounded in $E^{1}(\Omega)$ and tends as $\varepsilon \rightarrow 0$ to the global attractor $\mathcal{A}_{0}$ of the 'averaged' equation (7.2). Finally, under the generic assumption that all of the equilibria of equation (7.2) are hyperbolic, the global attractor $\mathcal{A}_{\varepsilon}$ is regular if $\varepsilon \leq \varepsilon_{0}$ (thanks to Theorem 5.3) and tends to the limit attractor $\mathcal{A}_{0}$ as $\varepsilon \rightarrow 0$ in the following sense

$$
\operatorname{dist}_{E^{1}(\Omega)}^{\text {symm }}\left(\mathcal{A}_{\varepsilon}, \mathcal{A}_{0}\right) \leq C \varepsilon^{\kappa},
$$

for some positive $C$ and $\kappa$ (we recall that the considered nonlinearity, obviously, satisfies assumptions (1.28) and (1.29) and, thus, (7.4) follows from Corollary 5.6).

Remark 7.3. The uniqueness problems with equation (7.3) can be partially overcome using the so-called trajectory approach, see [8], [9], [28] and [32]. We recall that, under this approach, instead of the classical way of constructing a dynamical system in a phase space $E(\Omega)$ associated with equation (7.3) (which can be defined as a semigroup of multivalued maps only), one considers the so-called trajectory dynamical system

$$
T_{h}^{\varepsilon}: \mathcal{K}_{\varepsilon}^{+} \rightarrow \mathcal{K}_{\varepsilon}^{+}, \quad\left(T_{h}^{\varepsilon} u\right)(t):=u(t+h), \quad h \geq 0
$$

where the trajectory phase space $\mathcal{K}_{\varepsilon}^{+} \subset L^{\infty}\left(\mathbb{R}_{+}, E(\Omega)\right)$ is the set of all (properly defined) weak energy solutions of problem (7.3) endowed by the appropriate topol-

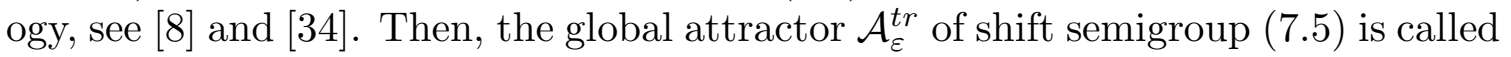
the trajectory attractor associated with problem (7.3) (it is also worth to emphasize that, in the case where we have the uniqueness, the trajectory dynamical system is usually equivalent to the classical one, see [8] and [34] for the details). 
The existence of trajectory attractors $\mathcal{A}_{\varepsilon}^{\text {tr }}$ for equations (7.3) and their weak convergence to the attractor $\mathcal{A}_{0}^{\text {tr }}$ of the limit equation (7.2) (which is equivalent to the global attractor $\mathcal{A}_{0}$, since we have the uniqueness for (7.2)) has been established in [9] and [10]. Moreover, as we proved in [34], every complete bounded weak solution $\xi_{u}(t), t \in \mathbb{R}$, of equation (7.3) belonging to the attractor $\mathcal{A}_{\varepsilon}^{t r}$ becomes regular as $t \rightarrow-\infty\left(\xi_{u}(t) \in E^{1}(\Omega)\right.$ if $t$ is small enough) and tends to the set $\mathcal{R}_{\varepsilon}$ of the equilibria of equation (7.3) (in a fact this result holds for every $\varepsilon>0$ ). Since the set $\mathcal{R}_{\varepsilon}$ is uniformly bounded in $E^{1}(\Omega)$ as $\varepsilon \rightarrow 0$ then we have the following result: for every complete weak solution $\xi_{u}(t)$ of equation (7.3), there exists time $T=T_{u}$ such that

$$
\left\|\xi_{u}(t)\right\|_{E^{1}(\Omega)} \leq C, \quad \forall t \leq T
$$

where the constant $C$ is independent of $\xi_{u}$ and $\varepsilon \leq \varepsilon_{0}$. Combining this result with Corollary 2.6 and arguing as in [34], we then establish that $\xi_{u}(t) \in E^{1}(\Omega)$ for every $t \in \mathbb{R}$ if $\varepsilon>0$ is small enough (i.e. such that $R_{0}(\varepsilon)>C$ ). Therefore, we proved that in this case

$$
\mathcal{A}_{\varepsilon}^{\text {tr }} \subset L^{\infty}\left(\mathbb{R}_{+}, E^{1}(\Omega)\right)
$$

and is uniformly bounded (with respect to $\varepsilon \rightarrow 0$ ) in this space. Thus, the trajectory attractor $\mathcal{A}_{\varepsilon}^{\text {tr }}$ which describes the long-time behavior of weak solutions of equation (7.3) with $\xi^{0} \in E(\Omega)$ coincides with the attractor $\mathcal{A}_{\varepsilon}$ constructed in Theorem 3.2, to be more precise, we have the relation

$$
\mathcal{A}_{\varepsilon}=\left.\mathcal{A}_{\varepsilon}^{t r}\right|_{t=0}, \quad \varepsilon \leq \varepsilon_{0} .
$$

Therefore, every weak energy solution $\xi_{u}(t), t \geq 0$ of equation (7.3) with arbitrary initial data $\xi^{0} \in E(\Omega)$ (and not necessarily $\xi^{0} \in B_{R_{0}(\varepsilon)}$ ) is attracted as $t \rightarrow+\infty$ by the global attractor $\mathcal{A}_{\varepsilon}$ constructed in Theorem 3.2.

The next example shows that equation (2.3) may have additional complete bounded solutions outside of the ball $B_{R_{0}(\varepsilon)}$ for every $\varepsilon>0$.

Example 7.4. Let us consider the following semilinear hyperbolic equation in a smooth bounded domain $\Omega \subset \mathbb{R}^{3}$ :

$$
\partial_{t}^{2} u+\gamma \partial_{t} u-\Delta_{x} u+u^{3}\left(1+\nu \cos \left(\frac{2 t}{\varepsilon}\right)\right)-\bar{a} u=0,\left.\quad \xi_{u}\right|_{t=\tau}=\xi^{\tau},\left.\quad \partial_{n} u\right|_{\partial \Omega}=0
$$

where $\bar{a} \in \mathbb{R}$ and $\nu>0$ is a small parameter. The averaged equation for (7.9) is, obviously, has the form of (7.2) with $g=0$.

Then, thanks to Corollary 2.6 and Theorem 3.2, for every $\xi^{\tau} \in B_{R_{0}(\varepsilon)}$, equation (7.9) possesses a unique global bounded solution $\xi_{u}(t), t \geq \tau$, and the dynamical processes generated by this equation on the ball $B_{R_{0}(\varepsilon)}$ possess the uniform attractors $\mathcal{A}_{\varepsilon}$ which are uniformly (with respect to $\varepsilon \rightarrow 0$ ) bounded in $E^{1}(\Omega)$ and tend to to the global attractor $\mathcal{A}_{0}$ of the averaged equation (7.2) in the sense of the upper semicontinuity in $E^{1}(\Omega)$.

Moreover, under the additional generic assumption that all of the equilibria of (7.2) are hyperbolic, the corresponding pullback attractors $\mathcal{A}_{\phi_{\varepsilon}}(\tau)$ are regular (due to Theorem 5.3) and we have estimate (7.4) for the symmetric distance between $\mathcal{A}_{\varepsilon}$ and $\mathcal{A}_{0}$ (due to Corollary 5.6 and Lemma 1.6).

Nevertheless, as shown in the next lemma, equation (7.9) possesses additional complete bounded solutions outside of the ball $B_{R_{0}(\varepsilon)}$ which tend to infinity as $\varepsilon \rightarrow 0$. 
Lemma 7.5. For all sufficiently small $\nu>0, \varepsilon>0$ and $\varepsilon \ll \nu$ equation (7.9) possesses at least one spatially homogeneous time-periodic solution $u_{\nu, \varepsilon}(t)$ (of period $2 \pi \cdot \varepsilon)$ which satisfies the following estimates:

$$
C_{1} \varepsilon^{-2} \leq\left\|\xi_{u_{\nu, \varepsilon}}(t)\right\|_{E(\Omega)}=\left\|\xi_{u_{\nu, \varepsilon}}(t)\right\|_{E^{1}(\Omega)} \leq C_{2} \varepsilon^{-2}
$$

where the positive constants $C_{1}$ and $C_{2}$ are independent of $\varepsilon$ and $\nu$.

Proof. Making the change of variables $z=t / \varepsilon$ and $v(z)=\varepsilon u(t / \varepsilon)$ in equation (7.9), we have:

$$
\partial_{z}^{2} v+\varepsilon \gamma \partial_{z} v-\varepsilon^{2} \Delta_{x} v+v^{3}(1+\nu \cos (2 z))-\varepsilon^{2} \bar{a} v=0
$$

Recall that we seek for the spatially homogeneous solutions of (7.11) only, so we may forget about the Laplacian and obtain the second order ODE:

$$
V^{\prime \prime}(z)+V^{3}(z)(1+\nu \cos (2 z))+\varepsilon \gamma V^{\prime}(z)-\varepsilon^{2} \bar{a} V(z)=0
$$

Moreover, since $u(t)=\varepsilon^{-1} V(t / \varepsilon)$, then for proving the lemma, it is sufficient to construct the $2 \pi$-periodic solution of (7.12) of order $O(1)$ as $\varepsilon \rightarrow 0$. In order to do so, we first construct a $2 \pi$-periodic solution $V_{\nu}(z)$ of the equation

$$
V_{\nu}^{\prime \prime}(z)+V_{\nu}^{3}(z)(1+\nu \cos (2 z))=0
$$

which corresponds to the case $\varepsilon=0$ in (7.12). Then, if the constructed solution is nondegenerate (e.g., hyperbolic), it preserves under small perturbations of equation (7.13) and, in particular, (7.12) possesses a limit cycle close to $V_{\nu}(z)$ if $\varepsilon \ll \nu$ is small enough. Thus, it only remains to find a hyperbolic $2 \pi$-periodic solution of equation (7.13) for small positive $\nu$.

It is worth to recall now that equation (7.13) is a Hamiltonian system with $3 / 2$ degrees of freedom (see e.g., [1], [2], [7], [25] and references therein). In particular, it is known that the desired periodic solution $V_{\nu}(z)$ can be computed in the form of power series with respect to the parameter $\nu$ :

$$
V_{\nu}(z)=V_{0}(z)+\nu V_{1}(z)+\nu^{2} V_{2}(z)+\cdots
$$

where $V_{i}(z)$ are the appropriate $2 \pi$-periodic functions which can be found by the standard recursive procedure. In particular, $V_{0}(z)$ should satisfy the conservative Hamiltonian equation (7.13) with $\nu=0$ :

$$
V_{0}^{\prime \prime}(z)+V_{0}^{3}(z)=0
$$

Thus, we first need to find the $2 \pi$-periodic solution $V_{0}(z)$ of equation (7.15). To this end, we endow equation (7.15) by the initial conditions $V_{0, L}(0)=L$ and $V_{0, L}^{\prime}(0)=0$ where $L>0$ is a parameter and denote the obtained unique solution of equation (7.15) by $V_{0, L}(z)$. Then, it is not difficult to verify, using the explicit integral formula for the solutions of (7.15) that $V_{0, L}(z)$ is $T$-periodic where

$$
T=T(L)=C L^{-1}
$$


and the constant $C>0$ is independent of $L$. In particular, fixing $L=L_{0}:=C /(2 \pi)$, we obtain the desired $2 \pi$-periodic solution $V_{0}(z)=V_{0, L_{0}}(z)$ of equation (7.15). We recall that, in a fact, we have the one-parametric family $\left\{V_{0}(z+h)\right\}_{h \in \mathbb{R}}$ of $2 \pi$ periodic solutions of equation (7.15), consequently, following the general procedure, in order to determine $h$, we should consider the so-called Poincaré integral, which has the form

$$
P(h)=-\int_{0}^{2 \pi} V_{0}(t+h)^{3} \cos (2 z) V_{0}^{\prime}(z+h) d z=2 \int_{0}^{2 \pi} V_{0}(z+h)^{4} \sin (2 z) d z
$$

see [25]. We claim that $P(0)=0$. Indeed, the function $z \rightarrow V_{0}(z)^{4}$ is even with respect to $z$ and $z \rightarrow \sin (2 z)$ is odd, consequently, the function $V_{0}^{4}(z) \sin (2 z)$ is odd and its mean equals zero. The analogous reasons show that

$$
P^{\prime}(0)=8 \int_{0}^{2 \pi} V_{0}(z)^{3} V_{0}^{\prime}(z) \sin (2 z) d z=4 \int_{0}^{2 \pi} V_{0}(z)^{4} \cos (2 z) d z>0
$$

Indeed, due to the symmetries $V_{0}(z) \rightarrow-V_{0}(z)$ and $V_{0}(z) \rightarrow V_{0}(-z)$ of equation (7.15) and our assumption for the initial data, we see that the function $\left[V_{0}(z)\right]^{4}$ is $\pi$-periodic, has a unique zero on the interval $[0, \pi]$ at $z=\pi / 2$ and is symmetric with respect to $z \rightarrow \pi-z$. This allows to rewrite (7.18) as follows:

$$
P^{\prime}(0)=16 \int_{0}^{\pi / 2}\left[V_{0}(z)\right]^{4} \cos (2 z) d z=16 \int_{0}^{\pi / 4}\left(\left[V_{0}(z)\right]^{4}-\left[V_{0}(\pi / 2-z)\right]^{4}\right) \cos (2 z) d z
$$

Since $V_{0}(z)$ is monotonically decreasing on $[0, \pi / 2]$ then $\left[V_{0}(z)\right]^{4}-\left[V_{0}(\pi / 2-z)\right]^{4}>0$ for $z \in[0, \pi / 4)$ which confirms that $P^{\prime}(0)>0$.

Thus, according to the general theory (see e.g., [25, ch.6 $\S 6]$ ), the power series (7.14) defines indeed a unique $2 \pi$-periodic solution of equation (7.13) (for small $\nu$ ) which tends to $V_{0}(z)$ as $\nu \rightarrow 0$. Thus, the desired solution $V_{\nu}(z)$ of equation (7.13) is constructed and it only remains to verify that this solution is non-degenerate. To this end, we use the standard expansions of the multipliers $\lambda_{1}$ and $\lambda_{2}$ of this solution

$$
\lambda_{1}=\alpha_{1} \nu^{1 / 2}+O(\nu), \quad \lambda_{2}=-\alpha_{1} \nu^{1 / 2}+O(\nu)
$$

where

$$
\alpha_{1}^{2}=\frac{1}{2 \pi K} P^{\prime}(0) \text { and } K=-\frac{\pi L_{0}^{4}}{\frac{d T}{d L}\left(L_{0}\right)}
$$

and the function $T=T(L)$ is defined by (7.16), see [25, ch. 6, §7].

In our case, we obviously have $K>0$ and, consequently, $\alpha_{1}^{2}>0$ and $\alpha_{1} \in \mathbb{R}$. Thus, due to (7.19), the limit cycle $V_{\nu}(z)$ is indeed hyperbolic for small $\nu>0$ and, consequently, equation (7.12) possesses a limit cycle close to $V_{\nu}(z)$ if $\varepsilon \ll \nu$ is small enough. Lemma 7.5 is proven.

Hence, thanks to Lemma 7.5, we have the time-periodic solution $u_{\nu, \varepsilon}(t)$ of equation (7.9) outside of the ball $B_{R_{0}(\varepsilon)}$ if $\nu>0$ and $\varepsilon \ll \nu$ are small enough. Moreover, estimates (7.10) show that this solution tends to infinity as $\varepsilon \rightarrow 0$. 
Remark 7.6. The existence of the solution $v_{\nu, \varepsilon}(z)$ in the last example is closely related with the so-called nonlinear parametric resonance phenomena which is typical for the hyperbolic equations (see [7], [30] and the references therein) and which is usually not observed in reaction-diffusion equations. Indeed, according to Lemma 7.5 the solution $u_{\nu, \varepsilon}(t)$ is close (for small $\varepsilon$ and $\nu$ ) to the solution $v_{0}(t):=1 / \varepsilon V_{0}(t / \varepsilon)$ of the following conservative equation:

$$
\partial_{t}^{2} v_{0}+v_{0}^{3}=0
$$

and the period $\pi \cdot \varepsilon$ of the parametrical exciting of system (7.9) differs by a factor two from the period $2 \pi \cdot \varepsilon$ of the internal oscillations in conservative system (7.21) (solution $v_{0}(t)$ ), which is typical for the parametric resonance phenomena.

We also note that, in contrast to the linear equation, the period of oscillations in (7.21) depends on its energy $E$ and decays as $E \rightarrow \infty\left(T_{\text {int }}=C E^{-1 / 2}\right)$ and this is the main reason why the solution $u_{\nu, \varepsilon}(t)$ tends to infinity as $\varepsilon \rightarrow 0$. Indeed, in order to compensate the energy decay provided by the dissipation term $\gamma \partial_{t} u$ by the energy income provided by the parametrical resonance, we need to have $T_{\text {int }} \sim T_{\text {par }}=\pi \cdot \varepsilon$ and, consequently, $E \sim \varepsilon^{-2}$ in complete agreement with (7.10).

It is worth noting here that Example 7.4 has an essential drawback, namely, we cannot construct the uniform attractor in the whole space $E^{1}(\Omega)$ for positive $\varepsilon$ (in fact, we do not know whether or not every solution equation remains bounded as $t \rightarrow \infty)$. In order to overcome this drawback, we conclude our exposition by the following modification of Example 7.4.

Example 7.7. Let us consider the following semilinear hyperbolic problem in the bounded two-dimensional domain $\Omega \subset \mathbb{R}^{2}$ :

$$
\partial_{t}^{2} u+\gamma \partial_{t} u-\Delta_{x} u+\varepsilon^{3} u^{5}+u^{3}(1+\nu \cos (2 t / \varepsilon))-\bar{a} u=0,\left.\xi_{u}\right|_{t=\tau}=\xi^{\tau}
$$

Since the two dimensional case can be considered as a particular case of the threedimensional one, as in Example 7.4 we have the global solvability of (7.22) for all $\xi^{\tau} \in B_{R_{0}(\varepsilon)}$, the existence of uniform attractors $\mathcal{A}_{\varepsilon}$ for the dynamical processes generated by this equation on the ball $B_{R_{0}(\varepsilon)}$ and their convergence as $\varepsilon \rightarrow$ to the global attractor of the limit equation (7.2) (with $g=0$ ) in the space $E^{1}(\Omega$ ). Moreover, the change of variables described in Lemma 7.5 in equation (7.22) gives the following equation:

$$
\partial_{t}^{2} v+\varepsilon \gamma \partial_{t} v-\varepsilon^{2} \Delta_{x} v+v^{3}(1+\cos (2 z))+\varepsilon v^{5}-\varepsilon^{2} \bar{a} v=0
$$

which is also close (for small $\varepsilon$ ) to equation (7.13). Therefore, the assertion of Lemma 7.5 remains valid for equation (7.22) as well.

On the other hand, the nonlinearity in equation (7.22) obviously satisfies estimate (6.1) (where the constant $C_{\delta}$ is not uniform with respect to $\varepsilon$ ), consequently, since every polynomial rate of growth is subcritical in the two-dimensional case (see, [3] and [29]), arguing in a standard way, we can verify that equation (7.22) is dissipative for all $\xi^{\tau} \in E(\Omega)$ for every fixed $\varepsilon>0$ and the corresponding dynamical process possesses a uniform attractor $\widetilde{\mathcal{A}_{\varepsilon}}$ which is a compact set of $E^{1}(\Omega)$. Nevertheless, due to the existence of a solution $u_{\nu, \varepsilon}(z)$ constructed in Lemma 7.5, this attractor does not coincide with $\mathcal{A}_{\varepsilon}$ :

$$
\widetilde{\mathcal{A}_{\varepsilon}} \neq \mathcal{A}_{\varepsilon}, \quad \forall \varepsilon \ll 1
$$


Thus, in contrast to the previous results on averaging of global and uniform attractors (see [14], [21] and references therein), we now have that, roughly speaking, the uniform attractor $\widetilde{\mathcal{A}_{\varepsilon}}$ consists of two basically different parts for small $\varepsilon$. The first part $\left(\mathcal{A}_{\varepsilon}\right)$ is regular, has large basin of attraction (which contains at least the ball $B_{R_{0}(\varepsilon)}$ with $R_{0}(\varepsilon) \rightarrow \infty$ as $\varepsilon \rightarrow 0$ ) and tends to the attractor $\mathcal{A}_{0}$ of the averaged equation as $\varepsilon \rightarrow 0$. In contrast to this, the irregular part which is provided by the parametric resonance phenomena (it is not empty due to Lemma 7.5) tends to infinity as $\varepsilon \rightarrow 0$. We belive that this picture is typical for the averaging of hyperbolic equations of the form (0.1) where the leading part of the nonlinearity contains the terms rapidly oscillating in time.

Remark 7.8. We note that it is not difficult to deduce from equation (7.11) and formula (7.19) for the multipliers that the instability index of the time periodic orbit $u_{\nu, \varepsilon}(t)$ tends to infinity as $\varepsilon \rightarrow 0$. Consequently, the fractal dimension of the attractor $\widetilde{\mathcal{A}_{\varepsilon}}$ of equation (7.22) also tends to infinity as $\varepsilon \rightarrow 0$ :

$$
\lim _{\varepsilon \rightarrow 0} \operatorname{dim}_{f} \widetilde{\mathcal{A}_{\varepsilon}}=\infty
$$

Moreover, it is also well known that, generically, equation (7.13) contains the socalled stochastic layers and chaotic hyperbolic sets inside of them, see e.g. [1]. Since any hyperbolic set preserves under the small perturbations, then we may also construct the chaotic hyperbolic set for equation (7.22). Thus, in contrast to the regular part of the attractor $\widehat{\mathcal{A}}_{\varepsilon}$ (the dynamic on which is close to the gradient dynamics of the averaged system, see Theorem 5.3) the dynamics on the irregular part of $\widehat{\mathcal{A}_{\varepsilon}}$ is usually chaotic.

\section{Appendix. Auxiliary estimates for the linear WAVE EQUation.}

In this appendix we formulate and prove some auxiliary results on the regularity of solutions of linear non-homogeneous wave equations which are essential for our study of averaging of the nonlinear equations. We start with the following standard result.

Proposition A.1. Let $u(t)$ be a solution of the following equation

$$
\partial_{t}^{2} u+\gamma \partial_{t} u-\Delta_{x} u+\lambda_{0} u=h(t), \quad \xi_{u}(0)=0,\left.\quad \partial_{n} u\right|_{\partial \Omega}=0
$$

in a smooth bounded domain $\Omega$ with positive constants $\gamma$ and. $\lambda_{0}$. Assume also that the right-hand side $h$ belongs to the space $L^{2}\left([0, T], H^{s}(\Omega)\right)$ for some $0 \leq s<3 / 2$ and $s \neq 1 / 2$. Then, the solution $\xi_{u} \in L^{\infty}\left([0, T], E^{s}(\Omega)\right)$ and the following estimate holds:

$$
\|u(t)\|_{H^{s+1}(\Omega)}^{2}+\left\|\partial_{t} u(t)\right\|_{H^{s}(\Omega)}^{2} \leq C \int_{0}^{t} e^{-\alpha(t-r)}\|h(r)\|_{H^{s}(\Omega)}^{2} d r,
$$

for some positive constants $C$ and $\alpha$.

Proof. Indeed, in the case $s=0,($ A.2) is a well-known energy estimate (see, e.g. [29]) and the general case $s \neq 0$ can be easily reduced to this particular one by applying the operator $\left(-\Delta_{x}\right)_{N}^{s / 2}$ to both parts of equation (A.1) (which can be 
done since $D\left(\left(-\Delta_{x}\right)_{N}^{s / 2}\right)=H^{s}(\Omega)$ for $0 \leq s<3 / 2$ and $s \neq 1 / 2$, see [31]) and Proposition A.1 is proven.

The next result, which gives the analogue of estimate (A.2) for the case where only the norm of $h$ in $H^{-1}\left([0, T], H^{s}(\Omega)\right)$ is known, is a basic technical tool in our averaging of semilinear hyperbolic equations.

Proposition A.2. Let $u(t)$ be a solution of equation (A.1) and let the external force $h$ satisfy the condition:

$$
\left\|\int_{t}^{t+\tau} h(z) d z\right\|_{H^{s}(\Omega)} \leq M, \quad \forall t, t+\tau \in[0, T], \quad \tau \in[0,1]
$$

where the exponent $s$ is the same as in Proposition A.1. Then, the following estimate holds:

$$
\|u(t)\|_{H^{s}(\Omega)}^{2}+\left\|\partial_{t} u(t)\right\|_{H_{N}^{s-1}(\Omega)}^{2} \leq C M^{2}
$$

where the constant $C$ is independent of $u, t$ and $h$.

Proof. Let us introduce a new unknown function $w(t)$ as follows:

$$
w(r):=\int_{0}^{r} e^{-\alpha(r-t)} u(t) d t, \quad \text { i.e., } \quad \partial_{t} w(t)+\alpha w(t)=u(t), \quad w(0)=0
$$

where $\alpha$ is some fixed positive number. Then, multiplying equation (A.1) by $e^{-\alpha(r-t)}$ integrating over $[0, r]$ and by parts and using that $\xi_{u}(0)=0$, we derive that this new function satisfies the following equation:

$$
\partial_{t}^{2} w+\gamma \partial_{t} w-\Delta_{x} w+\lambda_{0} w=\tilde{H}(t), \quad \xi_{w}(0)=0
$$

where $\tilde{H}(t):=\int_{0}^{t} e^{-\alpha(t-r)} u(r) d r$. We now note that equation (A.6) has the form of (A.1), consequently, due to Proposition A.1, we have the following estimate:

$$
\left\|\xi_{w}(t)\right\|_{E^{s}(\Omega)} \leq C^{\prime}\|\tilde{H}\|_{L^{\infty}\left([0, T], H^{s}(\Omega)\right)} \leq C^{\prime \prime} M .
$$

Moreover, expressing $\partial_{t}^{2} w$ from equation (A.6) and using (A.7), we derive that

$$
\left\|\partial_{t}^{2} w(t)\right\|_{H_{N}^{s-1}(\Omega)} \leq C_{1} M
$$

Since $u(t)=\partial_{t} w(t)+\alpha w(t)$ and $\partial_{t} u(t)=\partial_{t}^{2} w(t)+\alpha \partial_{t} w(t)$ then estimates (A.7) and (A.8) imply (A.4) and finishes the proof of Proposition A.2.

\section{REFERENCES}

1. V.Arnold and A.Avez, Ergodic problems of classical mechanics, W. A. Benjamin, Inc., New York-Amsterdam, 1968.

2. V.I.Arnold, Geometrical methods in the theory of ordinary differential equations, En. of math. Sci.,1, Springer-Verlag, Berlin, 1988.

3. A. V. Babin, M. I. Vishik, Attractors of Evolutionary Equations, North Holland, Amsterdam,, 1992.

4. A.Bensoussan, J.-L.Lions, and G.Papanicolaou, Asymptotic Analysis for Periodic Structures, N.-Holland, Amsterdam, 1978. 
5. N.N.Bogolubov, On some statistical methods in mathematical physics, Izd. AN USSR, Kiev, 1945.

6. A.Bourgeat and L.Pankratov, Homogenization of Semilinear Parabolic Equations in Domains with Spherical Traps, Appl. Anal. 64:3-4 (1997), 303-317.

7. M. Cartmell, Introduction to linear, parametric and nonlinear vibrations., Chapman and Hall, Ltd., London, 1990.

8. V.V.Chepyzhov and M.I.Vishik, Attractors for equations of mathematical physics, AMS, 2002.

9. V. Chepyzhov and M. Vishik, Evolution Equations and Their Trajectory Attractors, J. Math. Pures Appl. 76(10) (1997), 913-964.

10. V. Chepyzhov and M. Vishik, Perturbation of Trajectory Attractors for Dissipative Hyperbolic Equations, The Maz'ya annivesary collection 2 (Rostock 1998), 33-54.

11. M. V. Chepyzhov and M. Vishik, Averaging of trajectory attractors of evolution equations with rapidly oscillating terms, Sb. Math. 192 (2001), no. 1-2, 11-47.

12. H. Crauel and Flandoli, Attractors for random dynamical systems, Probab. Theory Relat. Fields 100 (1994), 1095-1113.

13. M. Efendiev and S. Zelik, Attractors of the Reaction-Diffusion Systems with Rapidly Oscillating Coefficients and their Homojenization, Ann. Inst. H.Poincare - AN 19 (2002), no. 6, 961-989.

14. M.Efendiev and S.Zelik, The regular attractor for the reaction-diffusion system with a nonlinearity rapidly oscillating in time and it's averaging, Adv. Differential Equations 8 (2003), no. 6, 673-732.

15. P.Fabrie, C.Galushinski, A.Miranville and S.Zelik, Uniform Exponential Attractors for a Singular Perturbed Damped Wave Equation, Disc. Cont. Dyn. Sys. 10 (2004), 211-238.

16. B. Fiedler and M.Vishik, Quantative averaging of global attractors of hyperbolic wave equations with rapidly oscillating coefficients. (Russian), Rus. Math. Survey 57 (2002), no. 4(345), 75-94.

17. B. Fiedler and M. Vishik, Quantitative homogenization of analytic semigroups and reactiondiffusion equations with Diophantine spatial frequencies, Adv. Differential Equations 6 (2001), no. 11, 1377-1408.

18. Yu. Goritski and M.I.Vishik, Integral manifolds for nonautonomous equation, Rend. Accad. Naz. XL, Mem. di Math. e Appl. 115 (1997), 106-146.

19. J.Hale, Asymptotic Behaviour of Dissipative Systems, Math. Surveys and Mon., 25(1987); AMS Providence, RI.

20. A.Haraux, Systemes dynamiques dissipatifs et applications, Paris, Masson, 1991.

21. A.Ilyin, Averaging for dissipative dynamical systems with rapidly oscillating righ-hand sides, Mat. Sbornik 187(5) (1996), 15-58.

22. P.Kloeden and B.Schmalfuss, Nonautonomous systems, cocycle attractors and variable timestep discretization, Numer. Algorthms 14 (1997), 141-152.

23. B.Levitan, Almost-periodic Functions, Gostekhizdat, Moscow, 1953.

24. B.Levitan and V.Zhikov, Almost-periodic Functions and Differential Equations, Moscov Univ. Press, Moscow, 1978.

25. I.G. Malkin, Some problems of the theory of nonlinear oscillations. (Russian), Gosudarstv. Izdat. Tehn.-Teor. Lit., Moscow, 1956.

26. Yu. Mitroploskii, The averaging method in nonlinear mechanics, Kiev, Naukova Dumka, 1971.

27. L.Pankratov and I.Chueshov, Homogenization of Attractors of Non-linear Hyperbolic Equations with Asymptotically Degenerate Coefficients, Sb. Math. 190:9 (1999), 1325-1352.

28. G. Sell, Global Attractors for the Three-Dimensional Navier-Stokes Equations, J. Dyn; Diff. Eqns 1 (1996), 1-33.

29. R.Temam, Infinite Dimensional Dynamical Systems in Mechanics and Physics, SpringerVerlag, New-York, 1988.

30. A. Tondl, T. Ruijgrok, F. Verhulst and R. Nabergoj, Autoparametric resonance in mechanical systems, Cambridge University Press, Cambridge, 2000.

31. H.Triebel, Interpolation Theory, Function Spaces, Differential Operators, N.-Holland, Amsterdam-New-York, 1978.

32. M. Vishik and S. Zelik, The trajectory attractor for a nonlinear elliptic system in an unbounded domain, Mat. Sbornik, 187, (1996), no. 12, 21-56. 
33. S. Zelik, The dynamics of fast nonautonomous travelling waves and homogenization, in Proc. of Conf. in Honor of R.Temam 60th annivesary, 7-10 of March 2000, Atlantique Editions de l'Actualite Scientifique, Poitou-Charents (2001), 131-142.

34. S.Zelik, Asymptotic regularity of solutions of singularly perturbed damped wave equations with supercritical nonlinearities, DCDS 11 (2004), no. 2-3, 351-392.

35. S.Zelik, Asymptotic regularity of solutions of a nonautonomous damped wave equation with a critical growth exponent, CPAA 3 (2004), no. 4, 921-934.

36. V.Zhikov, S.Kozlov, and O.Oleinik, Homogenization of Differential Operators and Integral Functionals, Springer-Verlag, Berlin, Heidelberg, New-York, 1994. 